\title{
JUNGBRONZEZEITLICHE FUNDE AUS VELIA
}

\author{
Einleitung $^{1}$
}

In den Jahren 1996/97 wurden auf der Akropolis von Velia (Südkampanien, Italien) am westlichen Ende der sog. Theaterterrasse archäologische Untersuchungen durchgeführt, die die Baugeschichte dieser Terrasse klären sollten. Die Terrasse wurde am Ende des ersten Viertels des 5. Jahrhunderts v. Chr. errichtet und überbaute Reste der spätarchaischen Wohnbebauung sowie eine massive Lehmziegelmauer, die möglicherweise im Zusammenhang mit einer ersten Befestigung der Akropolis zu sehen ist ${ }^{2}$. In den Straten, die bei der Aufgabe der Häuser und der Hinterfüllung der Stützmauer (Terrassenmauer I) entstanden sind, fanden sich in sekundärer Fundlage zahlreiche bronzezeitliche Keramikfragmente sowie Silexabschläge. Von besonderem Interesse war jedoch die Entdeckung einer ungestörten bronzezeitlichen Schicht (US 28) unter den spätarchaischen Häusern, durch welche erstmals in Velia ein Nutzungshorizont der Bronzezeit angeschnitten wurde (Abb. 1. 2). Insgesamt handelt es sich um 398 Keramikfragmente und 12 Silices. Sie stammen aus den US (unità stratigrafica) 3, 7, 11, 15, 16, 18, 24-30 und 383.

Bronzezeitliche Überreste wurden in Velia erstmals im Jahr 1966 von J.-P. Morel im Südbereich der Akropolis entdeckt, weshalb er auf dem westlichsten Sporn der Hügelkette von Castellamare della Bruca eine bronzezeitliche Besiedlung vermutete ${ }^{4}$. Allerdings stammten diese Funde alle aus gestörten Kontexten, vor allem aus den Planierungsschichten über den spätarchaischen Wohnhäusern bzw. aus Straten unterhalb dieser Strukturen ${ }^{5}$. Weitere bronzezeitliche Funde kamen bei den Grabungen von C. A. Fiammenghi auf der Akropolis im Südwesten des großen Tempels zutage ${ }^{6}$. Die Funde sind hier zahlreicher und charakteristischer als die aus der Grabung von 1966, stammen jedoch auch aus durchmischten Planierungsschichten, vor allem über den archaischen Wohnhäusern. Weitere Keramikfragmente konnten bei den österreichischen Grabungen am östlichen Ende der Theaterterrasse 1993 in den Schnitten 1/93 und 2/93 angetroffen werden (Abb. 3).

In den Grabungen von 1996/97 konnte nun erstmals bronzezeitliches Material aus einer ungestörten Schicht geborgen werden, welche den ersten geschlossenen Befund der Bronzezeit für Velia darstellt. Allerdings wurden auch hier keine Baustrukturen angetroffen, so daß der Beweis einer bronzezeitlichen Siedlung nicht erbracht werden konnte. Dennoch darf man angesichts der Art und Menge der Kleinfunde, die ausschließlich im Bereich der späteren Akropolis

\footnotetext{
1 Abkürzungen und Zitierweise folgen den ÖJh 69, 2000, 357 ff. (http://www.oeai.at/publik/autoren.html); zusätzlich s. u. die im Katalog verwendeten Abkürzungen.

2 F. Krinzinger - V. Gassner, Velia - neue Forschungen auf der Akropolis, ÖJh 66, 1997, 230-251.

3 Dem Leiter der österreichischen Ausgrabungen in Velia, F. Krinzinger, danke ich für die Möglichkeit, diesen Fundkomplex zu publizieren. V. Gassner danke ich für ihre stete Unterstützung bei der Durchführung dieser Arbeit.

4 J.-P. Morel, Vestiges de l'âge du bronze sur l'acropole de Velia, in: F. Krinzinger - B. Otto - E. Walde (Hrsg.), Forschungen und Funde. Festschrift B. Neutsch (1980) 299.

5 Morel (Anm. 4) 305. 306.

${ }^{6}$ C. A. Fiammenghi, Velia. Acropoli. Un saggio di scavo nell'area del tempio ionico, in: G. Greco - F. Krinzinger (Hrsg.), Velia. Studi e Ricerche (1994) 82.
} 


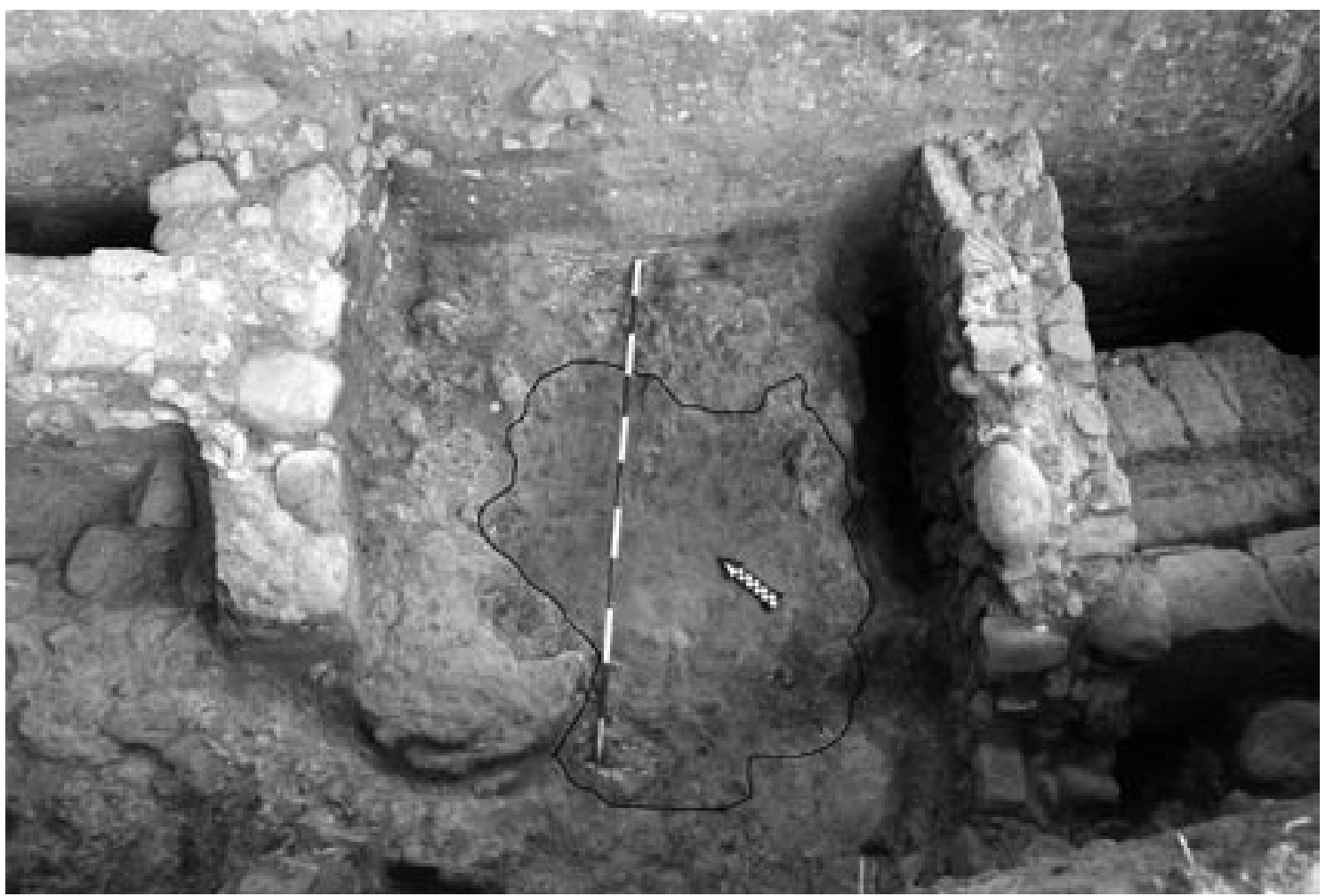

1 Blick auf die bronzezeitliche Schicht (US 28; unter Nordpfeil und Meßstange) in Schnitt 2/96

von Velia angetroffen wurden7, eine Besiedlung dieses Felssporns während der Bronzezeit für sehr wahrscheinlich halten. Sollte sich diese Annahme durch die weiteren Feldforschungen bestätigen, so kommt Velia aufgrund seiner Lage eine Sonderstellung unter den bronzezeitlichen Siedlungen in Südkampanien zu. Die bisher bekannten Siedlungen des Neolithikums und der Bronzezeit in jener Region liegen zwar nicht weit von der Küste entfernt, jedoch ohne unmittelbare Beziehung zum Meer ${ }^{8}$. Es handelt sich entweder um Freilandstationen oder um Höhlen. Die mutmaßliche Siedlung im Bereich der Akropolis von Velia hingegen befindet sich nicht auf der höchsten Erhebung der Hügelkette, sondern auf der dem Meer am nächsten liegenden. Bronzezeitliche Siedlungen in Süditalien mit vergleichbarer Lage sind Porto Saturo ${ }^{9}$ im Golf von Taranto und Coppa Nevigata ${ }^{10}$ an der adriatischen Küste.

Ein weiteres wichtiges Ergebnis der Grabungskampagne von 1996/97 in Velia ist, daß durch die Neufunde nun eine genauere chronologische und kulturelle Bestimmung des prähistorischen Materials innerhalb der Bronzezeit möglich war. Ordnete man die Keramik der Akropolis von Velia bisher allgemein der »appenninischen Bronzezeit « ${ }^{11}$ bzw. der »appenninischen Facies der mittleren Bronzezeit« ${ }^{12}$ mit einigen Belegen für die jüngere Bronzezeit zu, so läßt sich nach

\footnotetext{
7 Ein einziges Keramikfragment (Inv. 531/97-119, US 535), gefunden in der Unterstadt, dürfte aus diesem Bereich stammen, vgl. V. Gassner, Materielle Kultur und kulturelle Identität in Elea in spätarchaisch-frühklassischer Zeit, Velia Studia II, AForsch 8 (2003) 247.

8 L. Bernabò Brea, Gli Eoli e l'inizio dell'età del bronzo nelle isole Eolie e nell'Italia meridionale. Archeologia e leggende (1985) 188.

9 F. G. Lo Porto, VI. Satyrion (Taranto). Scavi e richerche nel luogo del più antico insediamento laconico in Puglia, NSc 18, 1964, 183 Abb. 3.

10 I. Damiani - M. Pacciarelli - A. C. Saltini, La facies archeologiche dell'isola di Vivara e alcuni problemi relativi al protoappenninico B, Annali di Archeologia e Storia Antica 6, 1984, Abb. 6, 23.

11 Morel (Anm. 4) 305.

12 Fiammenghi (Anm. 6) 84.
} 


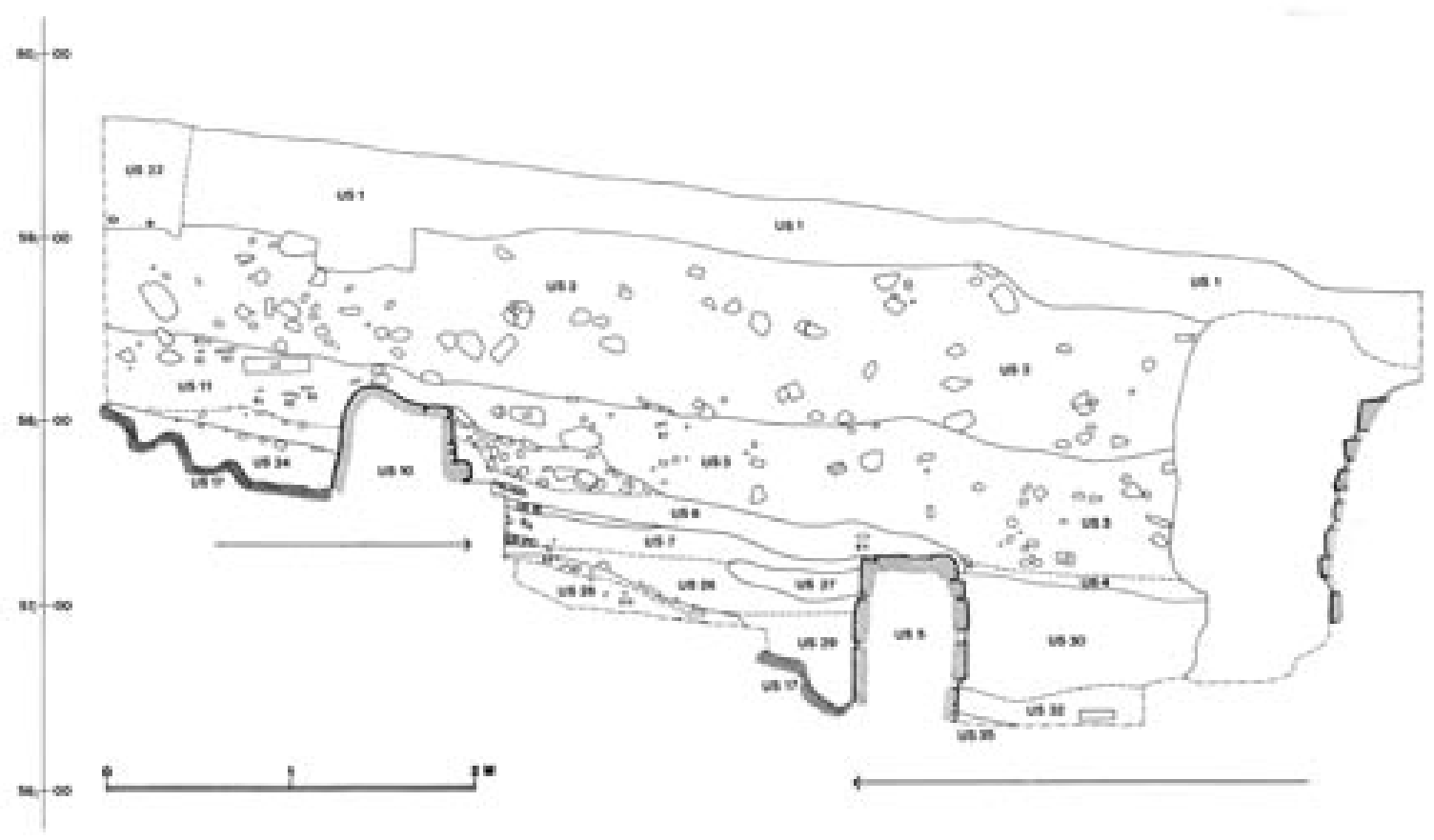

2 Nordostprofil des Schnittes 2/96. Bronzezeitliche Schicht (US 28) unterhalb der archaischen Besiedlung

Auswertung der neuen Funde und einer neuerlichen Interpretation der bereits veröffentlichen festhalten, daß das gesamte bekannte Material der subappenninischen Phase der Jungbronzezeit angehört.

Im ersten Teil des Beitrages werden die Funde aus den österreichischen Grabungen der Jahre 1993, 1996 und 1997 vorgelegt und ausgewertet. Bei ihrer chronologischen und kulturellen Einordnung im zweiten Teil wird auch die Keramik aus den Grabungen von J.-P. Morel und C. A. Fiammenghi herangezogen, soweit sie für die Datierung des Fundortes Velia relevant ist. Aufgrund der Nähe der drei Fundstellen und der Einheitlichkeit des Materials ist anzunehmen, daß sie zur selben Fundstelle gehörten.

\section{Charakterisierung des Fundmaterials}

\subsection{Die Keramik}

\subsubsection{Erhaltungszustand}

Der Zerscherbungsgrad der Gefäße ist sehr hoch. Es gibt kein einziges Gefäß, das sich vollständig rekonstruieren läßt. Von den meisten Gefäßen hat sich ein einziger Scherben erhalten, von einem konnte ein Drittel erfaßt werden (Taf. 2, 8). Die prozentuelle Erhaltung einzelner Gefäße ist ebenfalls gering und betrifft stark zerscherbte Keramik, wie beispielsweise das Gefäß auf Tafel 3, 5. Der hohe Zerscherbungsgrad der Keramik liegt hauptsächlich in der nicht besonders entwickelten Technologie begründet - geringe bzw. zu hohe Brenntemperaturen sowie allgemein die Verwendung von grob gemagerten Tonen. Dazu kommt weiters die häufige Umlagerung des Materials.

Der Abrollungsgrad der Scherbenbrüche verteilt sich von gezackt bis abgerundet, wobei der Hauptanteil bei den wenig abgerundeten bzw. abgerundeten liegt. Der unterschiedliche Abrollungsgrad der Scherben innerhalb derselben Schicht oder eines einzigen Gefäßes kann auf verschiedene technologische Faktoren sowie unterschiedliche Verhältnisse der Bodenlagerung und 


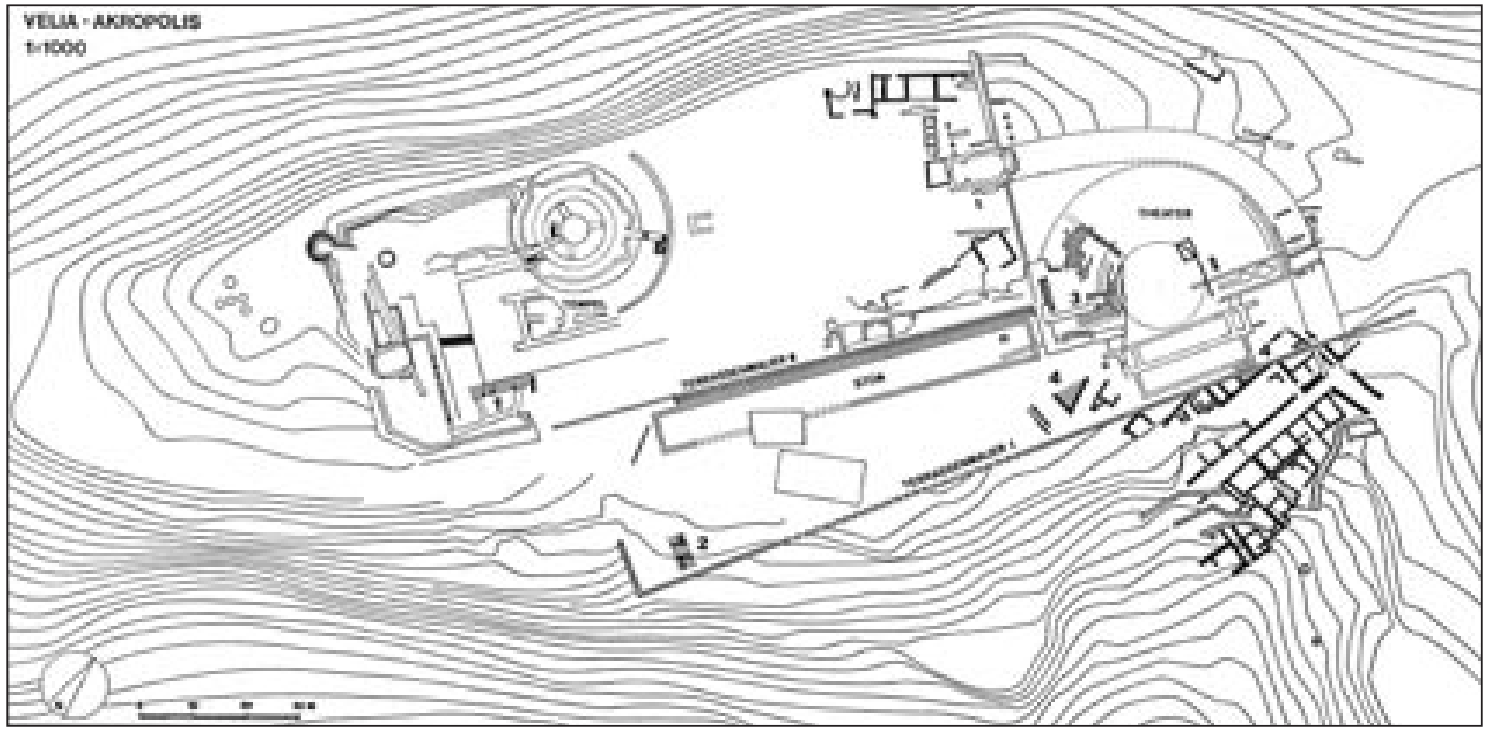

3 Die Akropolis von Velia. Bereiche mit bronzezeitlichem Fundmaterial: 1) Grabung C. A. Fiamminghi;

2) Grabung F. Krinzinger und V. Gassner 1996/97; 3) Grabung F. Krinzinger 1993; 4) Grabung J.-P. Morel

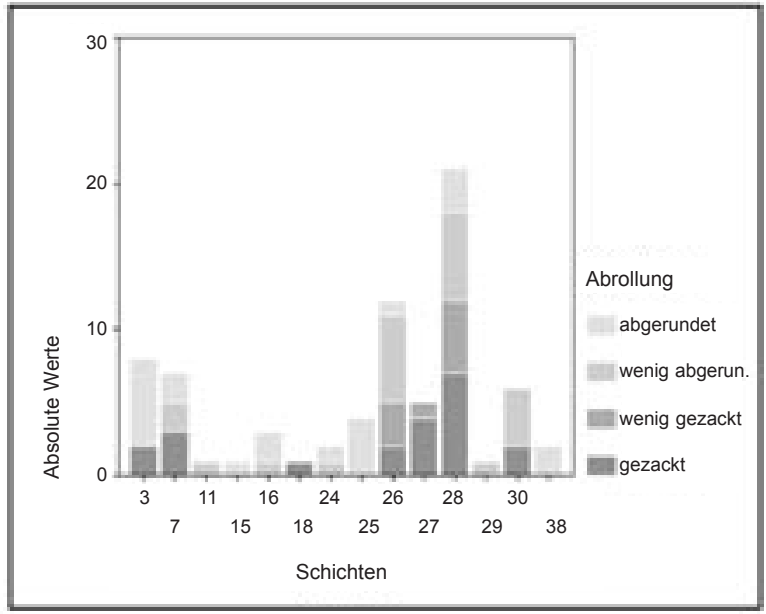

4 Verteilung der Abrollung der Keramik nach Schichten

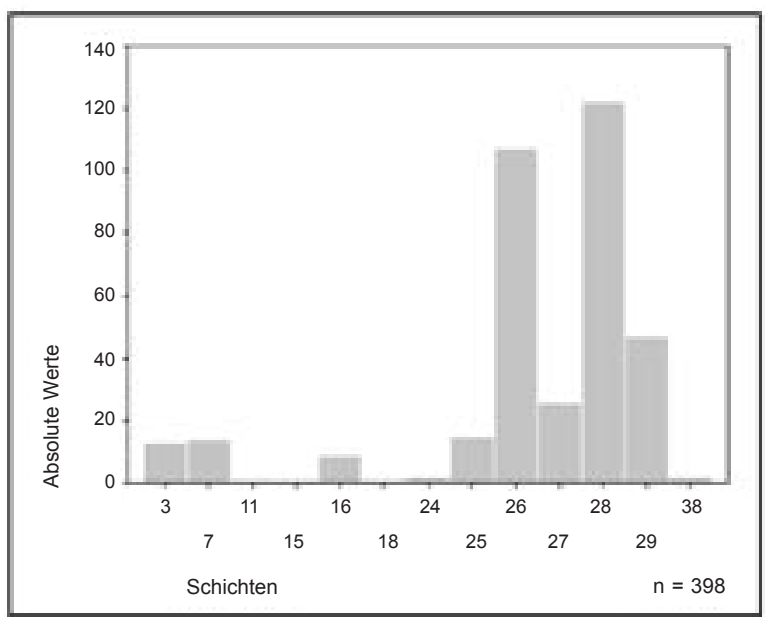

5 Verteilung der Keramik nach Schichten der physischen Erosionsprozesse zurückgeführt werden. So kann zwischen den Scherben der ungestörten bronzezeitlichen Schicht US 28 und jener der unmittelbar darüberliegenden Schichten US 25, 26 und 27 ein gewisser Unterschied festgestellt werden. Keramikfragmente mit gezackten bzw. weniger gezackten Brüchen kamen häufiger in den Schichten 26, 27 und 28 vor als in den spätarchaischen Schichten (US 3 , $7,18,30)$ bzw. waren hier gar nicht vorhanden (US 11, 15, 16, 24, 25, 29, 38) (Abb. 4). Der Verteilung der Abrollungsgrade entsprechen auch die Resultate der vertikalen Verteilung bronzezeitlicher Funde durch die Schichten. In den unteren Schichten waren mehr Gefäße aus der Bronzezeit vorhanden als in den oberen (Abb. 5). Diese Ergebnisse bestätigen die Beobachtungen der Stratigraphie ${ }^{13}$ : durch wiederholte Eingriffe in die unterste, direkt über dem anstehenden Fels liegende bronzezeitliche Kulturschicht während der griechischen Zeit gelangten bronzezeitliche Funde aus dem ursprünglichen Kontext bis zu den Zerstörungsschichten der spätarchaischen Häuser sowie bis in die obersten Grabungsschichten.

13 Krinzinger - Gassner (Anm. 2) 250. 


\subsubsection{Technologische Aspekte}

1.1.2.1 Materialcharakteristika

Die Charakteristika der Keramikmasse wurde z. T. makroskopisch, z. T. mit einer achtfach vergrößernden Handlupe erfaßt. Naturwissenschaftliche Analysen der Scherben wurden nicht durchgeführt. Mit einer einfachen Beschreibung einiger der physikalischen Eigenschaften der Scherben wurde versucht, Zusammenhänge zwischen der Struktur der Keramikmasse und der Gefäßform zu erkennen sowie technologische Aspekte zu erfassen. Die Kriterien der Beschreibung der Keramikmasse waren die Korngröße und -menge, die Verteilung der Körner, das Aussehen des Bruches sowie die Härte der Gefäßwände. Mit Ausnahme des letzten Merkmals wurden alle Eigenschaften am frischen Bruch des Gefäßes beobachtet. Es wurden fünf unterschiedliche Materialgruppen individualisiert und in Subgruppen differenziert, die von sehr feinkörnig bis zu grobkörnig reichen (Tab. 1). Die Subgruppierung des Materials beruht vor allem auf den unterschiedlichen Werten der Härte. Da die Brennbedingungen der Mehrheit der Gefäße nach der Beurteilung des Farbspektrums mehr oder weniger einheitlich erscheinen, können die Unterschiede der Härte in Zusammenhang mit den verwendeten Rohstoffen und Magerungen bzw. mit der Bodenlagerung stehen. $\mathrm{Ob}$ die Einschlüsse im Ton natürlich sind oder bei der Tonaufbereitung beigefügt wurden, konnte jedoch nicht eruiert werden. Die gleichmäßige Kornverteilung der Mehrheit der Gefäße könnte auf eine natürliche Herkunft hindeuten.

Die Mehrzahl der Gefäße gehört drei Materialgruppen an (Abb. 6): der fein- (2), der mittelfein- (3) und der grobkörnigen (4) Gruppe. Ein einziger Scherben läßt sich der Kategorie der geschlämmten Keramikmasse (1) zuweisen (Taf. 1,2). Ebenso kann nur ein Scherben als sehr grobkörnig (5) angesprochen werden (Taf. 4, 10). Fragmente von 14 Gefäßen wurden aus einer feinkörnigen Keramikmasse hergestellt. Sie haben in der Regel Körner bis $1 \mathrm{~mm}$ Größe. Einige Fragmente weisen vereinzelte Körner mit einer Größe bis zu 2 mm auf (z. B. Taf. 1, 4; 3, 4; 4, 6). Dennoch überwiegen große Körner im allgemeinen nicht. Bei feinkörnigen Gruppen mit geringerer Kornmenge ist das glatte Aussehen des frischen Bruches üblich (Taf. 1, 4; 2, 5. 6; 3, 5. 6. 7. 9; 4, 1. 3. 6. 8). Feinkörnige Scherben mit mittlerer (Taf. 1, 6; 3, 8) oder reichlicher (Taf. 3, 4) Kornmenge und mit körnigem Aussehen des Bruches (Taf. 1, 6; 2, 7; 3, 4. 8) bilden die Ausnahme. Scherben aus sechs Gefäßen haben eine grobe Keramikmasse mit vielen mittelgroßen, regelmäßig verteilten Körnern und einem körnigen Aussehen des Bruches (Taf. 1, 9; 2, 1. 2. 3; 4, 4; Inv. 225/96-15.1). Die Mehrheit der mittelkörnigen Gefäße hat eine gleichmäßig verteilte Magerung (Taf. 1, 5; 2, 4; 3, 1. 2. 3; Inv. 180/93-2.1, 203/96-155.2-3, 215/96-62.1, 228/96-4.13-14, 229/96-69.1, 247/93-3.1, 2028/97-2.4-5, 2038/97-10.1). Bei 18 Gefäßen konnte eine grobkörnige Keramikmasse festgestellt werden (Taf. 1, 1. 3. 7; 2, 8. 9; 3, 10; 4, 2. 4. 7; Inv. 224/96-15.1, 227/96-26.1, 228/96-4.5-6, 228/96-5.1-2, 261/96-2.1-2, 261/96-2.3, 2054/971.1, 2060/97-1.1-3, 2060/97-1.4-5). Die Körner verteilen sich ebenfalls regelmäßig, das Aussehen des Bruches ist dementsprechend körnig, und der Härtegrad variiert von normal bis sehr hart.

Die Korrelation von Materialgruppen und Gefäßformen läßt einen klaren Zusammenhang erkennen. Der Gruppe der feinkörnigen Keramik gehören vor allem kleine Formen an, wie die stark oder weich profilierten Knickwandschüsseln mit und ohne Henkel (Taf. 3, 5. 6. 7. 8), Schüsseln mit weicher S-Profilierung und ausladendem Rand (Taf. 3, 4) sowie vereinzelt kleine dünnwandige Töpfe (Taf. 1, 6) mit sehr gut geglätteten bzw. hoch polierten Oberflächen. Sie bilden die Gruppe der feineren Keramik. Solche Formen wurden nicht aus mittel- oder grobkörnigen Materialien hergestellt.

Aus mittelkörnigen Tonen wurden vier Kategorien von Gefäßen erzeugt: Schalen bzw. kleine Schüsseln mit gewölbter Wand und ausladendem Rand (Taf. 3, 1. 3; 4, 4), Schalen mit ausladendem Rand (Taf. 3, 2), klein- bis mittelgroße Töpfe mit niedrigem, ausladendem Hals (Taf. 1, 5; 1, 9) und Töpfe mit Fingerleistenverzierung (Taf. 2, 1. 2. 3. 4).

Grobkörnige Tone wurden für die Produktion von kleinen bis mittelgroßen Töpfen bevorzugt (Taf. 1, 3. 7; 2, 8). Eine einzige Schüssel mit großen Dimensionen (Taf. 3, 10), die daher als Vorratsgefäß interpretiert wird, und das 'oggetto fetillo' (Taf. 2, 9) weisen ebenfalls eine grob- 
Tabelle 1: Charakterisierung der Materialgruppen

\begin{tabular}{|c|c|c|c|c|c|}
\hline $\begin{array}{l}\text { Material- } \\
\text { gruppe }\end{array}$ & Korngröße & $\begin{array}{l}\text { Kornmenge } \\
\left(\text { pro } 1 \mathbf{c m}^{2}\right)\end{array}$ & $\begin{array}{c}\text { Verteilung der } \\
\text { Magerungsbestand- } \\
\text { teile }\end{array}$ & Härte & Bruch \\
\hline 1 & geschlämmt $(2,04 \mathrm{~g} / \mathrm{ccm})$ & wenig $(<$ als 5$)$ & sehr gleichmäßig & weich & glatt \\
\hline 2.1 & feinkörnig (bis $1 \mathrm{~mm}$ ) & mittel $(5-10)$ & gleichmäßig & hart & glatt \\
\hline 2.2 & $\begin{array}{l}\text { feinkörnig ( } 1 \mathrm{~mm} \text {, verein- } \\
\text { zelt } 2 \mathrm{~mm})\end{array}$ & wenig $(<$ als 5$)$ & gleichmäßig & normal & körnig \\
\hline 2.3 & $\begin{array}{l}\text { feinkörnig ( } 1 \mathrm{~mm} \text {, verein- } \\
\text { zelt } 2 \mathrm{~mm})\end{array}$ & wenig $(<$ als 5$)$ & ungleichmäßig & sehr hart & glatt \\
\hline 2.4 & feinkörnig (bis $1 \mathrm{~mm}$ ) & wenig $(<$ als 5$)$ & sehr ungleichmäßig & normal & glatt \\
\hline 2.5 & feinkörnig (bis $1 \mathrm{~mm}$ ) & mittel $(5-10)$ & gleichmäßig & hart & körnig \\
\hline 2.6 & feinkörnig (bis $1 \mathrm{~mm}$ ) & mittel $(5-10)$ & gleichmäßig & hart & glatt \\
\hline 2.7 & feinkörnig (bis $1 \mathrm{~mm}$ ) & viel $(10-20)$ & gleichmäßig & hart & körnig \\
\hline 2.8 & feinkörnig (bis $1 \mathrm{~mm}$ ) & viel $(10-20)$ & gleichmäßig & sehr hart & glatt \\
\hline 3.1 & mittelkörnig (bis $2 \mathrm{~mm}$ ) & mittel $(5-10)$ & gleichmäßig & hart & körnig \\
\hline 3.2 & mittelkörnig (bis $2 \mathrm{~mm}$ ) & viel $(10-20)$ & gleichmäßig & normal & körnig \\
\hline 3.3 & mittelkörnig (bis $2 \mathrm{~mm}$ ) & viel (10-20) & gleichmäßig & sehr hart & körnig \\
\hline 3.4 & mittelkörnig (bis $2 \mathrm{~mm}$ ) & mittel $(5-10)$ & gleichmäßig & klingend hart & körnig \\
\hline 4.1 & grobkörnig (bis $3 \mathrm{~mm}$ ) & mittel $(5-10)$ & gleichmäßig & normal & körnig \\
\hline 4.2 & grobkörnig (bis $3 \mathrm{~mm}$ ) & mittel $(5-10)$ & gleichmäßig & hart & körnig \\
\hline 4.3 & grobkörnig (bis $3 \mathrm{~mm}$ ) & mittel $(5-10)$ & gleichmäßig & sehr hart & körnig \\
\hline 4.4 & grobkörnig (bis $3 \mathrm{~mm}$ ) & viel $(10-20)$ & gleichmäßig & normal & körnig \\
\hline 4.5 & grobkörnig (bis $3 \mathrm{~mm}$ ) & viel (10-20) & gleichmäßig & hart & körnig \\
\hline 5 & sehr grobkörnig $(>3 \mathrm{~mm})$ & sehr viel ( $>$ als 20$)$ & sehr ungleichmäßig & klingend hart & körnig \\
\hline
\end{tabular}

körnige Tonmasse auf. Weiters gehören die kreisförmigen Tonplatten (Taf. 4, 9; Inv. 227/96-26.1, 228/96-5.1-2) zu dieser Materialgruppe.

Die Gefäße sind in der Regel - unabhängig von Form und Größe - hart gebrannt. Sehr stark oder schwach gebrannte Gefäße sind selten. Als Ausnahme kann die Härte der kreisförmigen Tonplatten angesprochen werden; sie sind weniger hart gebrannt als die Gefäße. Die Tonplatten wurden aus groben, sehr porösen und brüchigen Keramikmassen hergestellt.

\subsubsection{Gefäßaufbau}

Mit Ausnahme einer kleinen Anzahl von Scherben wurde die Keramik ausschließlich mit der Hand geformt. Sieben Gefäße wurden auf einer langsamen Drehscheibe hergestellt (Taf. 1, 1. 3. 6; 3, 1. 3; Inv. 224/96-15.1, 253/96-14.1-5, 259/96-4.3-4). An der Innen- und Außenseite dieser Gefäße sind umlaufende Spuren verwischter Fingerabdrücke zu sehen. Obwohl sie nicht so klar wie bei der eisenzeitlichen oder römischen Keramik zu beobachten sind, kann ihre Entstehung nur auf eine mechanische Drehbewegung zurückgeführt werden. Außerdem unterscheiden sich diese Gefäße von den übrigen auch dadurch, daß es sich ausschließlich um kleine Töpfe und Schalen handelt. Sie sind alle dickwandig, haben überwiegend eine dunkelbraune bis dunkelrote Tonfarbe und eine grobkörnige, brüchige Keramikmasse. Aufgrund der Charakteristika der Herstellungsmaterialien sind die Brüche gezackt.

\subsubsection{Wandstärke}

Die Wandstärke der Gefäße läßt sich in vier Gruppen unterteilen. Dabei sind gewisse Zusammenhänge zwischen Wandstärke und keramischer Klasse bzw. Gefäßtyp und zwischen Wandstärke und Gefäßgröße bzw. Materialgruppe zu beobachten. Gefäße mit einer Wandstärke zwi- 
schen 3.5 und $7 \mathrm{~mm}$ gehören der feinkörnigen keramischen Gruppe an. Eine Ausnahme stellt ein einziger Topf dar (Taf. 1, 7): er wurde aus einem grobkörnigen Material hergestellt und ist trotzdem dünnwandig.

$\mathrm{Zu}$ den dickwandigen Gefäßen mit einer Wandstärke zwischen 9.5 und 13 sowie $14 \mathrm{~mm}$ zählen fast allen Topftypen, ausgenommen die Gefäßtypen 8 und 9, sowie einige mittelgroße Schüsseln (Taf. 1, 1; 4, 8). Diese Wandstärke wies auch die Mehrheit der Scherben auf, die aufgrund ihres hohen Zerscherbungsgrades nicht einer bestimmten Gefäßform zugewiesen werden konnte. Genauso wurde für die Mehrheit

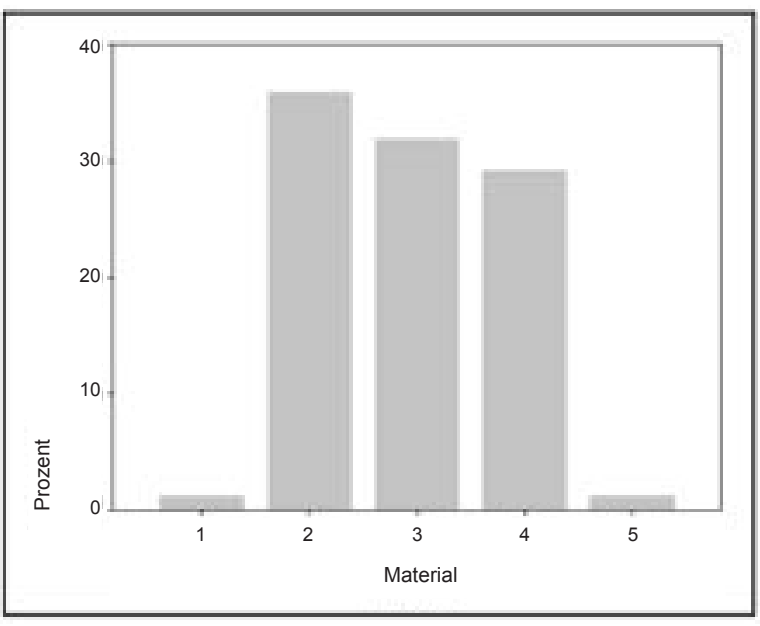

6 Häufigkeit der Materialgruppen der Töpfe ein grobkörniges bzw. mittelgrobkörniges Material verwendet. Daher ist es möglich, daß sie zu mittelgroßen oder großen Töpfen gehörten.

Eine Wandstärke von 16.5 und 18.5-40 mm wurde bei Fragmenten von drei Gefäßen beobachtet (Taf. 1, 4; 3, 10; Inv. 2028/97-3.1-2). Sie gehören zu zwei Töpfen und einer Schüssel, die aufgrund ihrer Größe als Vorratsgefäße bezeichnet werden.

Wegen der spezifischen Form und großen Dimensionen weisen die kreisförmigen Tonplatten die höchsten Wandstärken zwischen 20 und $26 \mathrm{~mm}$ auf.

\subsubsection{Oberfläche}

Die Oberfläche der Mehrheit der Gefäßscherben wurde außen und innen gut geglättet. Dies trifft vor allem auf mittel- bis dickwandige Gefäße zu. Mit Ausnahme einer Knickwandschüssel (Taf. 3, 6), die zur Feinware gehört, handelt es sich bei ihnen um Töpfe (Taf. 1, 8; 2, 1. 2. 3. 4. 7). Spatelartige Glättspuren auf der inneren Gefäßwand (Taf. 2, 8; 4, 4) oder Fingerspuren (Taf. 2, 6; 4, 10) sind selten. Letztgenannte sind nur auf einem Vorratsgefäß und einer Tonplatte erhalten.

Einige Gefäße, vor allem Schüsseln, wurden außerdem im lederharten Zustand so hochpoliert, daß ihre Oberfläche heute noch ein glänzendes Aussehen hat. Sie gehören hauptsächlich der feinkörnigen Materialgruppe an (Taf. 1, 6; 3, 4. 5. 6. 7. 8). Die Oberflächen zweier Töpfe aus mittelkörnigem Material wurden ebenfalls in dieser sorgfältigen Weise behandelt (Taf. 1, 5. 9).

Unregelmäßige Oberflächen sind selten. Sie beschränken sich auf ein Henkelgefäß mit Tupfenleistenverzierung (Taf. 4, 7), möglicherweise ein Vorratsgefäß, und ein Fragment eines nicht näher definierbaren Gegenstandes (Taf. 2, 9). Auch die Tonplatten zeigen unregelmäßige Oberflächen. Die Oberseite wurde mit der Hand grob geglättet, während die Unterseite sehr unregelmäßig ist. Der unregelmäßige Unterteil entstand dadurch, daß die Tonplatten während der Formgebung auf den Boden gelegt wurden.

\subsubsection{Farbe}

Die Farbe von 72 Gefäßen wurde an der Oberfläche und am frischen Kern bestimmt (Abb. 7). $41 \%$ der zu rekonstruierenden Gefäße weisen hell- bis dunkelrote bzw. hell- bis dunkelorange Oberflächen auf (Abb. 7: 1). Bei 12 ist der Kern rotbraun, dunkel- bis hellrot oder hellorange, bei den übrigen braun, grau oder schwarz. $40 \%$ aller Gefäße zeigen an beiden Oberflächen verschiedene Brauntöne mit mittel- bis dunkelbraunem, grauem oder schwarzem Kern (Abb. 7: 2). Nur bei $19 \%$ der Gefäße sind beide Oberflächen hell- bis dunkelgrau, der Kern dunkelgrau bis schwarz (Abb. 7: 3).

Im allgemeinen wurde die Keramik oxydierend gebrannt, wobei aber immer wieder reduzierende Brennphasen vorkamen. Die braune Farbe der Keramik deutet generell auf einen un- 


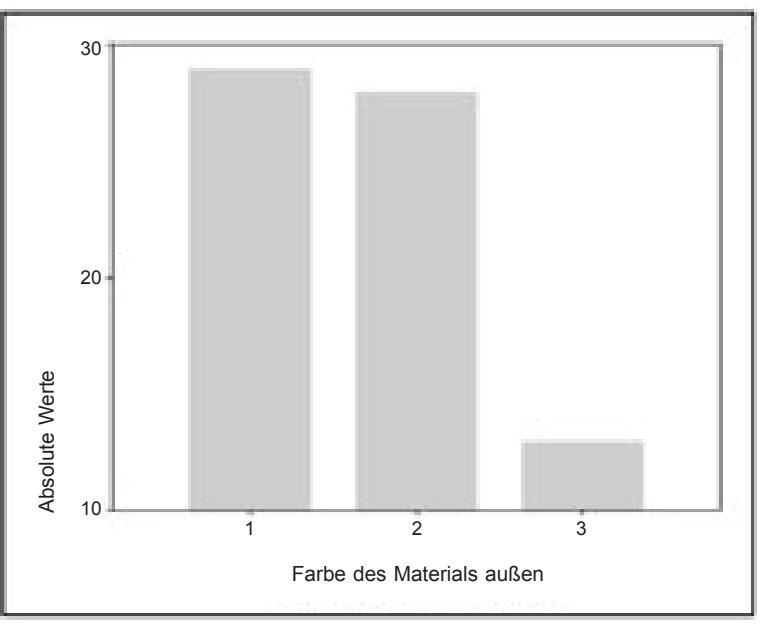

7 Häufigkeit der Farbe der äußeren Oberfläche vollständig oxydierenden Brand hin. Sie kann aber auch in einem oxydierenden Brandproze $ß$ beibehalten werden, wenn die natürliche Farbe des Tons braun war ${ }^{14}$. Ein vollständig reduzierender Brand konnte nur bei wenigen Gefäßen beobachtet werden.

Die Farbe der Oberfläche verteilt sich ohne erkennbare Normen auf die verschiedenen Gefäßtypen und -größen. Nur bei zwei Gefäßgruppen konnten Regelmäßigkeiten festgestellt werden: Die Oberfläche der Drehscheibenkeramik ist meist dunkelrot oder braun, jene der Vorratsgefäße mit Tupfenleistenverzierung hauptsächlich hellgelbbraun oder orange.

\subsubsection{Klassifikation der Keramikformen}

Aufgrund des hohen Zerscherbungsgrades der Gefäße konnte nur bei 130 von 398 Fragmenten die Gefäßform erfaßt werden. Insgesamt könnten 72 Gefäße bestimmt werden. Von diesen wurden 30 Gefäße und vier Henkel anhand von Vergleichen mit besser erhaltener Keramik aus verschiedenen Fundstellen in Süditalien bestimmten Formen zugeordnet. Die Kriterien dieser Klassifikation wurden aus dem Verhältnis zwischen Form, Proportionen und Durchmesser bestimmt. Dabei haben sich drei Formklassen ergeben - Töpfe, Schalen und Schüsseln, die sich in 16 Gefäßtypen unterteilen ließen.

\subsubsection{Töpfe}

- Gefäßtyp 1a: Kleiner Topf mit kugeligem Bauch und flachem Boden (14 cm BdDm) (Taf. 2, 8). Ein leicht nach innen eingezogener Rand vervollständigte den kugelförmigen Körper des Gefäßes, wie gleiche Exemplare aus der Nekropole von Copezzato ${ }^{15}$ und Grotta a Male ${ }^{16}$ zeigen.

- Gefäßtyp 1b: Mittelgroßer Topf mit gewölbter Schulter (Taf. 2, 7). Bei diesem Fragment handelt es sich vermutlich um den Schulterteil eines Gefäßes mit derselben Form wie Gefäßtyp 1a. Es ist aber größer ( $30 \mathrm{~cm}$ RdDm, $54 \mathrm{~cm} \mathrm{BaDm}$, ca. $33 \mathrm{~cm} \mathrm{H}$ ). Diese Maßangaben entsprechen einem Gefäß aus der Höhle Felci di Capri ${ }^{17}$.

- Gefäßtyp 2a: Kleiner Topf mit gerader Wand (Taf. 2, 4).

- Gefäßtyp 2b: Mittelgroßer Topf mit gerader Wand (Taf. 2, 1. 2; 4, 1. 2. 3, Inv. 2028/97-2.45, auch in den Grabungen von C. A. Fiammenghi ${ }^{18}$ und J.-P. More ${ }^{19}$ belegt). Obwohl die Exemplare dieses Gefäßtyps sehr fragmentiert sind, war seine morphologische Bestimmung möglich, weil sie immer mit einer charakteristischen Tupfenleistenverzierung versehen sind. In den mittel- bis spätbronzezeitlichen Fundstellen Süditaliens fanden sich zwei Grundformen in drei Größen: ein leicht doppelkonisches Gefäß mit hohem, fast geradem Oberteil,

14 Vgl. A. Shepard, Ceramics for the Archaeologist, Carnegie Institution of Washington, Publication 609 (1976) 105.

15 A. Mutti - E. Pellegrini, La necropoli di Copezzato ed i riti funerari degli 'Italici terramaricoli': un riesame, Bullettino di Paletnologia Italiana 86, 1995, 352 Abb. 17, 2; 358 Abb. 20, 2.

16 S. Panutti, Gli scavi di Grotta a Male presso l'Aquila, Bullettino di Paletnologia Italiana 78, 1969, 189 Abb. 22 , 3.

17 A. Marzocchela, L'eneolitico a Napoli, in: G. Macchiaroli (Hrsg.), Napoli antica I (1985) 34 Taf. III-3.11.

18 Fiammenghi (Anm. 6) 83 Abb. 118-119, 1-2.

19 Morel (Anm. 4) 305 Abb. 21. 
leicht eingezogenem Unterteil und flachem Boden sowie ein Gefäß mit gewölbter Wand, ausladendem Rand und ebenfalls flachem Boden. Sie sind in drei Großkategorien $-16 / 37 / 50 \mathrm{~cm}$ RdDm, 17/37/40 cm BaDm, 20/34/60 cm H - in Grotta del Noglio ${ }^{20}$, Grotta a Male ${ }^{21}$, Coppa Nevigata ${ }^{22}$, Punta Mezzogiorno di Vivara ${ }^{23}$ üblich. Dieser Gefäßtyp konnte auch mit Lappenhenkeln versehen werden (Taf. 2, 6) und ist auch aus der Grabungskampagne von J.-P. Morel belegt ${ }^{24}$. Die Henkel wurden paarig und waagrecht vorwiegend am Rand oder auf die Gefäßmitte gelegt, wie Beispiele aus dem oberen und unteren Niveau der Quadranten D und E und oberem Stratum der Quadranten A und B von S. Maria in Castello ${ }^{25}$ zeigen. Ein weiteres spezifisches Merkmal dieses Gefäßtyps ist die regelhaft dicke Wandstärke, die mittelkörnige Tonmasse und die überwiegend hellgelbbraunen bzw. orange Farben.

- Gefäßtyp 3: Mittelgroßer Topf mit eingezogenem Oberteil (14 cm RdDm; Taf. 1, 7. 8). Diese Gefäße sind als doppelkonische Töpfe mit flachem Boden zu ergänzen ${ }^{26}$.

- Gefäßtyp 4: Mittelgroßer, weitmundiger Topf mit ausladender Wand und abgerundeter Lippe $(22 \mathrm{~cm}$ RdDm; Taf. 1, 9). Auch an anderen Fundstellen konnte diese Gefäßform nicht vollständig erfaßt werden. Töpfe dieses Typus können einen Randdurchmesser bis zu $32 \mathrm{~cm}$ erreichen, wie ein Exemplar aus Grotta a Male zeigt ${ }^{27}$.

- Gefäßtyp 5: Großer, weitmundiger Topf mit ausladender Wand und stark profilierter Lippe (Taf. 1, 4). Wie der Gefäßtyp 4 gehört auch dieser Typus zu den Töpfen mit großem Randdurchmesser: ein ähnliches Gefäß aus Grotta a Male weist einen Randdurchmesser von $48 \mathrm{~cm}^{2} \mathrm{auf}^{28}$.

- Gefäßtyp 6: Kleiner Topf mit geöffnetem Mundzaun, gerader Wand und nach außen geknicktem Rand (16 cm RdDm; Taf. 1, 5). Ein etwas größeres Gefäß aus dem Niveau 4c der Grotta a Male ${ }^{29}$ ähnelt dieser Form sehr, wodurch das Exemplar aus Velia als Topf identifiziert werden konnte.

- Gefäßtyp 7a: Mittelgroßer Topf mit gewölbter Schulter und nach außen geknicktem Rand (18 cm RdDm; Taf. 1, 1). Solche Formen findet man in der Siedlung S. Maria in Castello ${ }^{30}$; sie konnten in Casa Lopez als Ganzform beobachtet werden. Es handelt sich um einen Topf mit doppelkonischem Bauch und abgerundetem Umbruch; der Rand ist nach außen geknickt, der Boden flach. Auf der Schulter weist er kleine runde Knubben auf, am Bauchumbruch zwei gegenständig angesetzte Henkel ${ }^{31}$.

- Gefäßtyp 7b: Mittelgroßer Topf mit gewölbter Wand und nach außen geknicktem Rand (Taf. 1, 3). Ähnliche Ränder werden häufig in Fundstellen des terramaren Gebietes gefunden. Normalerweise weisen sie einen kugelförmigen Bauch und einen flachen Boden auf ${ }^{32}$.

- Gefäßtyp 8: Kleiner Bandhenkeltopf mit Trichterhals und gewölbter Schulter. Der obere Teil des Henkels endet in zwei kleinen, auseinanderstehenden Appendices (Taf. 1, 2). Der Ge-

20 A. Vigliardi, Il bronzo 'appenninico' della grotta del Noglio, Rivista di Scienze Preistoriche 30, 1975, 290 Abb. 6, 3; 291 Abb. 7, 2.

21 Pannuti (Anm. 16) 232 Abb. 49, 1.

22 S. M. Cassano - A. Cazzella - A. Manfrechini, Coppa Nevigata e il suo territorio. Testimonianze archeologiche dal VII al II millennio a.C. (1987) 149 Abb. 72, 1; 150 Abb. 73, 7.

23 Museo Nazionale di Napoli, sala di preistoria, Vitrine 8.

24 Morel (Anm. 4) 304. 305 Abb. 15. 16. 17.

25 A. Vigliardi - M. Ghezzi, Insediamento del bronzo a S. Maria in Castello, Rivista di Scienze Preistoriche 31/1, 1976, 167 Abb. 17, 2; 199 Abb. 25, 9.

26 Vigliardi - Grezzi (Anm. 25) 149 Abb. 8, 1; R. Peroni, Protostoria dell'Italia continentale. La Penisola italiana nelle età del bronzo e del ferro (1989) $62 \mathrm{Abb} .13,1.2 .3$.

27 Pannuti (Anm. 16) 189 Abb. 22, 7.

28 Pannuti (Anm. 16) 180 Abb. 17, 12.

29 Pannuti (Anm. 16) 160 Abb. 7, 3.

30 Vigliardi - Grezzi (Anm. 25) 153 Abb. 10, 16.

31 L. Bernabò Brea - M. Cavalier, Meligunìs Lipára VI. Filicudi - Insediamenti dell'età del bronzo (1991) 37 Abb. 11a. c.

32 Peroni (Anm. 26) 74 Abb. 19, 7. 
fäßtyp kommt mit gleichen Dimensionen, aber nie als Ganzform in zahlreichen Fundzusammenhängen vor, z. B. in Cortine Fabriano ${ }^{33}$, Coppa Nevigata ${ }^{34}$ und Felci di Capri ${ }^{35}$. Seine exakte Form und Größe konnten bis jetzt nicht näher bestimmt werden.

- Gefäßtyp 9: Kleiner Topf mit kurzem, ausladendem Rand und geradem Hals (Taf. 1, 6). Für dieses Gefäß konnte keine Parallele gefunden werden. Ähnlichkeiten zeigt ein Gefäß aus dem Niveau 4b von Grotta a Male ${ }^{36}$, das etwas größer ist. Möglicherweise gehört es zu den topfartigen Gefäßen aus feinkörnigem Ton.

\subsubsection{Schalen}

- Gefäßtyp 10: Kleine Schale mit gewölbter Wand und ausladendem Rand (Taf. 3, 1. 3). In der Siedlung Casa Lopez auf Lipari wurde eine sehr ähnliche Schale gefunden. Sie weist einen niedrigen Unterteil, einen flachen Boden und kleine Henkel auf ${ }^{37}$. Ihre Dimensionen sind am Beispiel der Schale aus dem Niveau 4b der Grotta Male zu erfassen - $28 \mathrm{~cm} \mathrm{RaDm}$, $24 \mathrm{~cm} \mathrm{BaDm}, 8 \mathrm{~cm} \mathrm{H}^{38}$.

\subsubsection{Schüsseln}

- Gefäßtyp 11: Große Schüssel mit gewölbter Wand und flacher Lippe (50 cm RdDm; Taf. 3, 10). Vergleichbare Ränder gehören zu großen Vorratsgefäßen, deren Randdurchmesser bis $70 \mathrm{~cm}$ erreichen $\mathrm{kann}^{39}$.

- Gefäßtyp 12a: Kleine Schüssel mit gewölbter Wand und flachem Boden (8 cm BdDm; Taf. 4, 4). Solche Böden gehören zu kleinen Schüsseln mit gewölbter Wand und leicht eingezogenem Rand (15 cm RdDm, $16.5 \mathrm{~cm} \mathrm{BaDm}, 7.5 \mathrm{~cm} \mathrm{H})$, wie ein Beispiel aus der Nekropole von Copezzato zeigt ${ }^{40}$.

- Gefäßtyp 12b: Kleine Schüssel mit leicht (Taf. 4, 3) oder stark (Taf. 4, 2) eingedelltem Boden (4-6 cm BdDm). Auch in der Nekropole von Copezzato wurde ein Gefäß mit selber Form und gleichen Dimensionen wie Gefäßtyp 12a (11.4 cm RdDm, $12 \mathrm{~cm} \mathrm{BaDm,} 8.1 \mathrm{~cm}$ $\mathrm{H})$ aufgefunden, aber mit eingezogenem Boden ${ }^{41}$. Böden mit einem stark ausgeprägten Omphalos gehören zu kleinen Schüsseln mit abgerundeter Wandung oder einem niedrig angesetzten Wandumbruch und besitzen in der Regel einen kurzen Bandhenkel (10-15 cm RdDm, 10.5-14 cm BaDm, 6.5-7.5 H) ${ }^{42}$.

- Gefäßtyp 13: Mittelgroße Schüssel mit gewölbter Wand (Taf. 4, 7). Ähnliche Schüsseln wurden aus dem oberen Niveau des Quadranten C und dem unteren Niveau der Quadranten $\mathrm{D}$ und $\mathrm{E}$ von S. Maria in Castello geborgen ${ }^{43}$.

- Gefäßtyp 14: Kleine Schüssel mit gewölbter Wand und ausladendem, verdicktem Rand (Taf. 3, 2). Unter den Beispielen von Casa Lopez findet sich diese Form mit niedrigem Körper und fast abgerundetem Boden ${ }^{44}$.

- Gefäßtyp 15a: Kleine Knickwandschüssel mit eingezogenem Oberteil und gewölbtem, außen leicht konkavem Unterteil (14 cm RdDm; Taf. 3, 6. 7. 8). Bei diesen Schüsseln kann der Umbruch hoch (Taf. 3, 7) oder in der Gefäßmitte liegen (Taf. 3, 6. 8). Sie weisen kleine

33 D. Lollini, Il bronzo finale nelle Marche, Rivista di Scienze Preistoriche 34/1-2, 1979, 181 Abb. 1, 8.

${ }_{34}$ Cassano - Cazzella - Manfrechini (Anm. 22) 161 Abb. 80, 5.

35 Marzocchella (Anm. 17) 32 Taf. II-3.9.

36 Pannuti (Anm. 16) 164 Abb. 9, 13.

37 Brea - Cavalier (Anm. 31) Taf. 11, 1.

38 Mutti - Pellegrini (Anm. 15) 164 Abb. 9, 5.

39 Pannuti - Pellegrini (Anm. 15) 208 Abb. 33, 8; 223 Abb. 43, 8.

40 Mutti - Pellegrini (Anm. 15) 350 Abb. 16, 1.

${ }_{41}$ Mutti - Pellegrini (Anm. 15) 329 Abb. 5, 1.

42 Mutti - Pellegrini (Anm. 15) 324 Abb. 2, 2; 325 Abb. 3, 2; 352 Abb. 17, 3.

43 Vigliardi - Grezzi (Anm. 25) 165 Abb. 16, 5; 186 Abb. 21, 7.

${ }_{44}$ Brea - Cavalier (Anm. 31) 35 Abb. 9j. 
Dimensionen auf, wie die Beispiele aus der Grotta a Male veranschaulichen - 14 und $16.5 \mathrm{~cm}$ RdDm, 14.8 und $18 \mathrm{~cm} \mathrm{BaDm}, 6.4$ und $7.5 \mathrm{~cm} \mathrm{H}^{45}$.

- Gefäßtyp 15b: Kleine Knickwandschüssel mit leicht eingezogenem Oberteil und über den Rand hochgezogenem Henkel; der Unterteil ist gewölbt und der Boden außen leicht eingedellt (13.6 cm BaDm, $3 \mathrm{~cm}$ BdDm; Taf. 3, 5). Diese Schüsselform ist dem Gefäßtyp 15a sehr ähnlich, weist aber einen höheren Unterteil auf. Eine identische Form mit ähnlichen Dimensionen kommt in der Siedlung S. Maria in Castello vor $(16 \mathrm{~cm} \mathrm{RaDm}, 16.4 \mathrm{~cm} \mathrm{BaDm}$, $6 \mathrm{~cm} \mathrm{BdDm}, 9.6 \mathrm{~cm} \mathrm{H})^{46}$. Die paarigen Henkel sind in gleichzeitigen Siedlungen relativ häufig zu finden: Cortine di Fabriano und Bachero di Cingoli - Marche/Ancona ${ }^{47}$, Coppa Nevigata $^{48}$ und Stratum Ausonio I der Akropolis von Lipari ${ }^{49}$.

- Gefäßtyp 16: Kleine, tiefe Schüssel mit gewölbter Wand und ausladendem Rand $(8 \mathrm{~cm}$ RdDm; Taf. 3, 4; Inv. 225/96-15.1). Sehr ähnliche Formen finden sich etwa in Hütte B von Leporano $^{50}$ und im Niveau $4 \mathrm{~b}$ von Grotta a Male ${ }^{51}$. Die letztgenannten Beispiele lassen sich auch in der Größe (9.2 cm RdDm, $9.2 \mathrm{~cm} \mathrm{BaDm}, 6 \mathrm{~cm} \mathrm{H})$ gut mit dem Gefäß von Velia vergleichen.

\subsubsection{Henkel}

- Typ lumaca oder cornuta (Taf. 4, 5): Diese und die zwei folgenden Henkelarten gehören zu mittleren bis großen Knickwandschüsseln und steigen über das Randniveau an. Parallelen zum Henkel vom Typ cornuta fand man im unteren und oberen Niveau der Quadranten A und B und im oberen Stratum des Quadranten C von S. Maria in Castello ${ }^{52}$, im Niveau 3 von Grotta a Male ${ }^{53}$ und in Cortine di Fabriano ${ }^{54}$.

- Typ cilindrica-retta doppia (Taf. 4, 6): Entsprechende Beispiele kommen in der Siedlung auf der Akropolis von Lipari ${ }^{55}$ vor, weiters im Niveau D der Siedlung Grotta di Baffoni ${ }^{56}$, oberes Niveau der Quadranten A und B von S. Maria in Castello ${ }^{57}$, Cortine di Fabriano ${ }^{58}$ und Stratum 3 des Quadranten 1 von Grotta a Male ${ }^{59}$.

- Bandhenkel (Taf. 4, 1): Vergleichsbeispiele finden sich in der Siedlung Fontevecchia di Camerano $^{60}$ und auf der Akropolis von Lipari ${ }^{61}$.

\subsubsection{Sonstige Tongegenstände}

- Tonplatte (20-30 cm RdDm; Taf. 4, 9. 10; Inv. 227/96-26.1, 228/96-5.1-2): Gute Vergleiche wurden im Niveau 3, Quadranten 3 und 5 der Grotta a Male angetroffen ${ }^{62}$.

45 Pannuti (Anm. 16) 180 Abb. 17, 8; 202 Abb. 30, 2.

46 Vigliardi - Grezzi (Anm. 25) 151 Abb. 9, 8; 203 Abb. 26, 14.

47 Lollini (Anm. 33) 181 Abb. 1, 20; 199 Abb. 6, 45.

48 Cassano - Cazzella - Manfrechini (Anm. 22) 160 Abb. 79, 12.

49 L. Bernabo Bréa - M. Cavalier, Meligunìs Lipára IV. L'acropoli di Lipari nella preistoria (1980) 705-709 Taf. 204, $1-2$.

50 D. Lollini, III. - Leporano (Taranto). - La stazione protostorica di Porto Perone, NSc 17, 1963, 299 Abb. 20. 21.

51 Pannuti (Anm. 16) 164 Abb. 9, 1.

52 Vigliardi - Grezzi (Anm. 25) 161 Abb. 14, 1-5; 181 Abb. 19, 8-9; 203 Abb. 26, 10. 12.13.

53 Pannuti (Anm. 16) 215 Abb. 38, 13.

${ }^{54}$ Lollini (Anm. 33) 183 Abb. 2, 2. 10.

55 L. Brea - M. Cavalier, Civiltà preistoriche delle isole Eolie e del territorio di Milazzo, Bullettino di Paletnologia Italiana 65/1, 1956, 69 Abb. 44a.

56 A. Radmilli, Richerche intorno la gola del Sentino. III: gli scavi nella grotta dei Baffoni, Bullettino di Paletnologia Italiana 65/2, 1956, 527 Abb. 3, 8.

57 Vigliardi - Grezzi (Anm. 25) 203 Abb. 11

58 Lollini (Anm. 33) 183 Abb. 2, 4.

59 Pannuti (Anm. 16) 205 Abb. 32, 7.

60 Lollini (Anm. 33) 206 Abb. 8, 27.

${ }_{61}$ Bréa - Cavalier (Anm. 49) Taf. 209, 2.

62 Pannuti (Anm. 16) 177 Abb. 16, 15; 194 Abb. 25, 15. 
- Sog. oggetto fetillo (Taf. 2, 9): Fragmente entsprechender Objekte fand man in Sizilien und auf den Äolischen Inseln in Kontexten der 'cultura milazzese', wie beispielsweise in den Siedlungen Piano del Porto (Filicudi), S. Ipolito di Caltagione (Sizilien) ${ }^{63}$ und der Siedlung Montegnola (Lipari) ${ }^{64}$. Von der Akropolis von Lipari stammt ein vollständiges Objekt, das die Erfassung von Form und Dimensionen ermöglichte: Es handelt sich um einen zylindrischen, gekrümmten, massiven Körper, der in einem etwas breiteren, leicht eingedellten oberen Teil endet. Die Basis ist kreisförmig und breit. Die Höhe des Objekts beträgt ungefähr $25 \mathrm{~cm}^{65}$. Derartige Gegenstände haben eine weite Verbreitung vom Schwarzen Meer über den gesamten Mittelmeerraum bis zur südspanischen Atlantikküste. Häufiger treten sie in der Ägäis und in Süditalien vor allem in eisenzeitlichen Kontexten auf. Da dieses Objekt der Form eines Phallus ähnelt, wird es in der Forschung mit Fertilitätsriten in Verbindung gebracht ${ }^{66}$. Gegenstände ähnlicher Form sind auch aus Siedlungsbefunden griechischer Zeit bekannt und werden als Stützen interpretiert, die paarig aufgestellt einen Kochtopf hielten ${ }^{67}$. Eine ähnliche profane Interpretation als Kochtopfstützen wäre auch für die älteren Beispiele denkbar.

\subsubsection{Keramikverzierung}

Von wenigen Ausnahmen abgesehen ist die Keramik unverziert. Die Verzierung kann als Abdruckverzierung oder plastisch ausgeführt werden.

Die Abdruckverzierung kann aus geraden, horizontalen oder kreisförmigen Rillen bestehen (Taf. 2, 7). Diese bildeten möglicherweise ein zusammengehöriges Motiv mit 2-4 horizontalen Streifen und 2-4 Halbbögen wie in Paduletto di Coltano ${ }^{68}$, Fontevecchia di Camerano ${ }^{69}$ und La Braglia $^{70}$. Die Motive waren auf der Schulter eines Topfes angebracht, wie ein ähnliches, aber besser erhaltenes Stück aus der Höhle Felci di Capri erkennen läßt ${ }^{71}$.

Einmalig ist die plastische Verzierung mit einer kleinen runden Knubbe (Taf. 1, 1). Sie sitzt auf der Schulter eines Topfes, wie das Beispiel von Casa Lopez zeigt.

Die häufigste Verzierungsart ist eine plastische dekorative Leiste, bei der sich zwei Varianten unterscheiden lassen: eine leicht (Taf. 4, 7) und eine stark (Taf. 2, 1-5; 4, 8) eingedrückte Leiste. Beide Verzierungsarten wurden mit dem Finger hergestellt. Der Unterschied liegt darin, daß bei der ersten der Tonwulst mit dem Finger so eingedrückt wurde, daß seine zylindrische Form bestehen blieb, während bei der zweiten der Tonwulst mit dem Finger stärker eingedrückt wurde, wodurch eine Wellenleiste entstand. Ähnliche Fragmente von der Akropolis von Velia aus der Nähe des archaischen Tempels ${ }^{72}$ und aus der Grotta del Noglio ${ }^{73}$ zeigen, daß diese Verzierung unmittelbar unterhalb des Randes und in der Mitte des Gefäßbauches angebracht war. Selten wurde sie auch am Gefäßunterteil in Bodennähe gesetzt.

${ }_{63}$ Bréa (Anm. 8) 112 Abb. 100a. b.

64 Bréa - Cavalier (Anm. 31) Taf. 103g-i.

${ }_{65}$ Bréa - Cavalier (Anm. 55) 63 Abb. 40h.

${ }_{66}$ Bréa (Anm. 8) 112.

67 S. Morris, $\Lambda$ A $\Sigma$ ANA: A contribution to the ancient Greek kitchen, Hesperia 44, 1985, 398.

68 P. Bagnoli - N. Panicucci, L'insediamento del Paduletto di Coltano nell'ambito dell'Italia centrale, Rassegna di Archeologia 10, 1991/92, 673 Abb. A1.

69 Lollini (Anm. 33) 206 Abb. 8, 28.

70 J. Tirabassi, La Braglia (Reggio Emilia): il nucleo centrale del sito, Rassegna di Archeologia 10, 1991/92, 655 Nr. 1.

71 Marzocchella (Anm. 17) 34 Taf. III-3.11.

72 Fiammenghi (Anm. 6) 83 Abb. 118. 119.

73 Vigliardi (Anm. 20) 291 Abb. 7, 2. 


\subsection{Die Silices}

Bei den 12 gefundenen Silices handelt es sich um unvollständige Abschläge, die wahrscheinlich als Werkzeuge gedient haben. Sie stammen großenteils aus dem bronzezeitlichen Stratum US 28. Bei fünf Exemplaren (Inv. 2028.6.1-5) ist die Farbe durch Hitzeeinwirkung rötlich verfärbt. Auf Reste eines Brandes weist auch eine größere Menge verkohlten Holzes in diesem Bereich.

\section{Chronologische Stellung}

Die Gliederung der europäischen Bronzezeit geht auf P. Reinecke zurück und wurde 1959 von H. Müller-Karpe auf das italienische Gebiet übertragen ${ }^{74}$. Die derzeit für Süditalien verwendete Chronologie wurde mit Hilfe der stratigraphischen Sequenzen von Siedlungen unter Einbeziehung einphasiger Siedlungen und Gräber erstellt; sie hängt aber stark von der allgemeinen Fundtypologie der Bronzezeit ab. Eine wichtige Rolle spielt dabei die Korrelation mit der Chronologie des ägäischen Raumes. Das ist möglich, da seit dem 16. Jahrhundert v. Chr. in vielen süditalienischen Fundstätten griechische Keramikimporte vorkommen ${ }^{75}$. Sie haben einerseits eine besondere Bedeutung, um die durch die Stratigraphie nur unvollständige relative Chronologie der bronzezeitlichen Funde Süditaliens zu ergänzen; andererseits können durch sie genauere Anhaltspunkte für eine chronologische Einordnung gewonnen werden, weil auch das Spektrum der ${ }^{14} \mathrm{C}$-Daten für Italien noch sehr spärlich ist ${ }^{76}$. Das Vorhandensein verschiedener chronologischer Schemata mit unterschiedlichen Bezeichnungen für denselben Zeitabschnitt, aber auch einer unterschiedlichen Trennung der zeitlichen Abschnitte ${ }^{77}$ zeigt, daß bis jetzt eine befriedigende Lösung für die bronzezeitliche Chronologie Süditaliens noch aussteht. Aus einem Versuch, einen Überblick zu bieten, entstand die Zeittabelle in Tabelle 2.

Die chronologische Einordnung des bronzezeitlichen Materials von Velia wird durch zwei Faktoren erschwert: den hohen Zerscherbungsgrad der Keramik und die Kontinuität bestimmter Merkmale während der gesamten Bronzezeit. Dies sind die Hauptgründe, warum J.-P. Morel und C. A. Fiammenghi die Keramik aus ihren Grabungen in die Mittel- bis Jungbronzezeit datiert haben. Die Grabungskampagne von 1996/97 erbrachte eine Zunahme charakteristischer Funde, die nun eine gesicherte Datierung in die Jungbronzezeit ermöglichen.

$\mathrm{Zu}$ den für diesen Zeitabschnitt charakteristischen Keramikformen gehören: der Topf mit nach außen geknicktem Rand und Knubben auf der Schulter (Taf. 1, 1), der Henkeltopf (Taf. 1, 2), der Topf mit leicht eingedellter Randlippe (Taf. 1, 3), der weitmundige Topf mit stark profilierter Lippe (Taf. 1, 4), die Töpfe mit eingezogenem Rand (Taf. 1, 7. 8), die Schale mit ausladendem Rand (Taf. 3, 1. 3), die Schüssel mit ausladendem, verdicktem Rand (Taf. 3, 2), die großen Schüsseln mit breiter und flacher Lippe (Taf. 3, 10), die Knickwandschüsseln mit Henkelappendices (Taf. 3, 5), der Henkel vom Typ cornuta (Taf. 4, 5), der Henkel vom Typ cilindrico-retta doppia (Taf. 4, 6), der einfache Henkel ${ }^{78}$ und der Bandhenkel (Taf. 4, 1).

Aus den älteren Grabungen können folgende Gefäße ebenso als jungbronzezeitlich angesprochen werden: Gefäße mit horizontalen plastischen Leisten ohne den charakteristischen Tupfenabdruck $^{79}$, Schüsseln mit Zipfelrand ${ }^{80}$, Töpfe mit kurzem, geradem Rand und gewölbtem

\footnotetext{
74 H. Müller-Karpe, Beiträge zur Chronologie der Urnenfelderzeit nördlich und südlich der Alpen, Römisch-Germanische Forschungen 22 (1959) 334.

75 Vgl. Peroni (Anm. 26) 241-245 Abb. 54-56.

76 Vgl. R. W. Ehrich, Chronologies in Old World Archaeology II² (1992) 234-235.

77 Vgl. Damiani - Pacciarelli - Saltini (Anm. 10) 1-38.

78 Fiammenghi (Anm. 6) 82 Abb. 117, 7; vgl. Bréa - Cavalier (Anm. 49) Taf. 201, 6; 205, 2; Lollini (Anm. 32 ) 181 Abb. 1, 13. 24. 25; Vigliardi - Ghezzi (Anm. 25) 181 Abb. 19, 1-7; 203 Abb. 26, 2-6.

79 Fiammenghi (Anm. 6) 83 Abb. 119, 3; Vigliardi - Ghezzi (Anm. 24) 153 Abb. 10, 20; 155 Abb. 11, 1. 2. 4. 5.

80 Morel (Anm. 4) 300 Abb. 6.
} 
Tabelle 2: Chronologie zur Mittel- und Jungbronzezeit in Süditalien und auf den Äolischen Inseln

\begin{tabular}{|c|c|c|c|c|}
\hline $\begin{array}{l}\text { Konventionelle } \\
\text { Chronologie }\end{array}$ & $\begin{array}{l}\text { Technologische } \\
\text { Stufe }\end{array}$ & Kultur/Facies & $\begin{array}{l}\text { Hauptfundorte in } \\
\text { Süditalien }\end{array}$ & $\begin{array}{c}\text { Hauptfundorte und } \\
\text { Kultur/Facies auf } \\
\text { den Äolischen } \\
\text { Inseln }\end{array}$ \\
\hline $\begin{array}{l}\text { 1700-1500 } \\
(\mathrm{MH} \text { III B-LH II A) }\end{array}$ & $\begin{array}{l}\text { Bronzo medio } \\
\text { iniziale } \\
\text { (Frühmittelbronze- } \\
\text { zeit) }\end{array}$ & $\begin{array}{l}\text { Facies Mezzogiorno } \\
\text { oder Protoappennini- } \\
\text { ca B1 }\end{array}$ & $\begin{array}{l}\text { Punta Mezzogiorno, } \\
\text { Sektor A (Vivara); } \\
\text { Punta Capitelle, } \\
\text { Sektor E }\end{array}$ & Kultur Milazzese \\
\hline $\begin{array}{l}\text { 1500-1400 } \\
\text { (LH II B-LH III A1) }\end{array}$ & $\begin{array}{l}\text { Bronzo medio un } \\
\text { poco avanzato } \\
\text { (Mittlere Phase der } \\
\text { Bronzezeit) }\end{array}$ & $\begin{array}{l}\text { Facies Punta } \\
\text { d'Alaca oder Pro- } \\
\text { toappenninica B2 }\end{array}$ & $\begin{array}{l}\text { Punta d'Alaca, west- } \\
\text { und Ostsektoren } \\
\text { (Vivara) }\end{array}$ & Kultur Milazzese \\
\hline $\begin{array}{l}1400-1300 \\
(\text { LH III A2) }\end{array}$ & $\begin{array}{l}\text { Bronzo medio } \\
\text { avanzato } \\
\text { (Spätere Phase der } \\
\text { Bronzezeit) }\end{array}$ & Facies appenninica & $\begin{array}{l}\text { Punta Capitello, } \\
\text { Sektor B (Vivara); } \\
\text { Castiglione d'Ischia; } \\
\text { Grotta del Felci }\end{array}$ & Kultur Milazzese \\
\hline $\begin{array}{l}\text { 1300-1200 } \\
\text { (LH II B1-B2) }\end{array}$ & $\begin{array}{l}\text { Bronzo recente } \\
\text { (Jungbronzezeit) }\end{array}$ & $\begin{array}{l}\text { Facies } \\
\text { sub- } \\
\text { appenninica }\end{array}$ & $\begin{array}{l}\text { Grotta del Noglio; } \\
\text { Riparo dello } \\
\text { Zachito; } \\
\text { Casalvelino } \\
\text { (alle Südkampanien) }\end{array}$ & $\begin{array}{l}\text { Facies Ausonio I: } \\
\text { Acropoli di Lipari; } \\
\text { Filicudi; } \\
\text { Panarea }\end{array}$ \\
\hline $\begin{array}{l}1200-1100 \\
(\text { LH III c) }\end{array}$ & $\begin{array}{l}\text { Bronzo finale } \\
\text { iniziale } \\
\text { (Frühendbronzezeit) }\end{array}$ & $\begin{array}{l}\text { Facies proto- } \\
\text { vilanoviana }\end{array}$ & $\begin{array}{l}\text { Starza; } \\
\text { Sant'Angelo; } \\
\text { Grotta di Polla, } \\
\text { Straten 1-3 }\end{array}$ & $\begin{array}{l}\text { Facies Ausonio II: } \\
\text { Castelo di Lipari } \\
\text { sud; } \\
\text { Monfalcone, via XX } \\
\text { Settembre }\end{array}$ \\
\hline
\end{tabular}

Bauch $^{81}$, Töpfe mit Bandhenkeln am Rand oder Bauch ${ }^{82}$, Henkel mit gefurchter Oberseite (ansa cristata $^{83}$ sowie die einfachen Henkel ${ }^{84}$. Die wenigen Fragmente, die im appenninischen Stil verziert sind ${ }^{85}$, stellen eine Degeneration der klassischen Form dar und können daher nur schwer mit der charakteristischen appenninischen Verzierung der Mittelbronzezeit verglichen werden ${ }^{86}$. Ähnlich verzierte Keramik wie die aus Velia wurde ebenfalls in subappenninischen Kontexten in Coppa Nevigata ${ }^{87}$ aufgefunden.

Ein weiterer wichtiger Faktor für die Einordnung unserer Funde in die Jungbronzezeit ist das Vorkommen von Drehscheibenkeramik. Sie ist nicht sehr zahlreich, hat in ihren Formen aber gute Parallelen in der jungbronzezeitlichen Keramik Süditaliens. Die Drehscheibenkeramik wird auf griechischen Einfluß zurückgeführt, der erst mit der Jungbronzezeit faßbar wird ${ }^{88}$. Auch Höhensiedlungen wurden in Süditalien erst ab der Jungbronzezeit angelegt.

Dagegen zeigen andere Gefäße Charakteristika, die seit dem Anfang der Mittelbronzezeit vorkommen, aber bis in endbronzezeitlichen Kontexte weiterleben. Dazu gehören die Töpfe mit kugeligem Bauch und flachem Boden (Taf. 3, 8), die Töpfe mit Tupfenleistenverzierung (Taf. 2,

81 Morel (Anm. 4) 300. 301 Abb. 4; vgl. Pannuti (Anm. 16) 181 Abb. 18, 12; 191 Abb. 23, 4.

82 Morel (Anm. 4) 302 Abb. 11; vgl. Pannuti (Anm. 16) 176 Abb. 15. 16, 17; 192 Abb. 24, 1. 4. 5.

83 Morel (Anm. 4) 303 Abb. 13; vgl. Pannuti (Anm. 16) 176 Abb. 15, 6. 13; 194 Abb. 25, 2.

${ }^{84}$ Fiammenghi (Anm. 6) 82 Abb. 117, 8; vgl. Cassano - Cazzella - Manfrechini (Anm. 22) 160 Abb. 79, 17-19.

85 Morel (Anm. 4) 301 Abb. 5; Fiammenghi (Anm. 6) 82 Abb. 117, 1-4.

${ }^{86}$ z. B. Felci di Capri: vgl. Marzocchela (Anm. 17) 32 Taf. II-3.15 und Vivara: vgl. I. Damiani - M. Marazzi - M. Pacciarelli, L'insediamento preistorico di Vivara, in: Macchiaroli (Anm. 17) 42 Taf. 4, 46. 48.

87 Cassano - Cazzella - Manfrechini (Anm. 22) 158 Abb. 77, 3; 160 Abb. 79, 2-5.

88 R. Peroni, Hauptzüge der demographischen Entwicklung in Italien anhand von Siedlungs- und Grabfunden, Internationale Archäologie 36, 1997, 224. 
1-5), die Lappenhenkel (Taf. 2, 6), die Knickwandschüssel (Taf. 3, 6-8), der Topf mit Rillenverzierung (Taf. 2, 7), die Schüssel mit flachem (Taf. 4, 4) oder eingedelltem Boden (Taf. 4, 2. 3) und die Tonplatten (Taf. 4, 9. 10). Da diese Funde gemeinsam mit einer reichen und unstrittig jungbronzezeitlichen Hinterlassenschaft aufgefunden wurden, sind sie wahrscheinlich ebenfalls jungbronzezeitlich.

Aus diesem zeitlichen Rahmen fallen zwei Objekte heraus. Es sind dies das sog. oggetto fetillo, das für die mittelbronzezeitliche Milazzese-Kultur der Äolischen Inseln und Nordostsiziliens typisch ist, sowie der Henkel mit gefurchter Oberseite (ansa cristata) ${ }^{89}$, der ein kennzeichnendes Element der Endbronzezeit darstellt. Er kann in vereinzelten Fällen aber auch schon in Kontexten der Jungbronzezeit vorkommen, wie die Beispiele von Bachero di Cingoli ${ }^{90}$, S. Maria in Castello ${ }^{91}$, Coppa Nevigata ${ }^{92}$ und Fontevecchia di Camerano ${ }^{93}$ zeigen. Singulär bleibt ein Steinbeil neolithischer Herstellungstechnik, das während der Grabungskampagne 2001 auf der Felsoberfläche unterhalb der Fundamente des Theaters aufgefunden wurde ${ }^{94}$.

Die besten Vergleiche für das Material aus Velia liefern die folgenden Siedlungen Süditaliens, die alle den Zeitstufen Bronzo recente, Facies sub- oder mesoappenninica zuzurechnen sind: Porto Saturo, Quadrant H, Stratum 195; Porto Perone, Hütte A und B, Stratum B (subappenninisch) und weniger aus dem Stratum E (spätappenninisch) ${ }^{96}$; Cortine de Fabriano, einphasige Siedlung ${ }^{97}$; Grotta a Male, Quadranten 2-5, Stratum 398; S. Maria in Castello, oberes Niveau des Quadranten C, unteres Niveau der Quadranten D und E und vor allem unteres Niveau der Quadranten $\mathrm{A}$ und $\mathrm{B}^{99}$; Coppa Nevigata, subappenninische und jungsubappenninische Phase ${ }^{100}$; Akropolis von Lipari auf den Äolischen Inseln, Stratum Ausonio I ${ }^{101}$. Als weitere Fundstellen aus Kampanien - allerdings ohne stratigraphische Unterteilung - sind Cività di Paterno ${ }^{102}$, Casalvelino ${ }^{103}$, Riparo dello Zachito ${ }^{104}$ und Pertosa ${ }^{105}$ anzuführen.

89 Morel (Anm. 4) 304 Abb. 14.

90 Lollini (Anm. 33) 199 Abb. 6, 6.

91 Vigliardi - Ghezzi (Anm. 25) 203 Abb. 26, 9.

92 Cassano - Cazzella - Manfrechini (Anm. 22) 160 Abb. 79, 20.

93 Lollini (Anm. 33) 206 Abb. 8, 11.

94 M. Steskal, Das Theater von Velia. Bericht über die Ausgrabungen 2001, ÖJh 71, 2002, 278 ff. bes. 283-284: Anhang 3: Ein neolithisches Steinbeil aus Velia (K. Rebay).

95 Lo Porto (Anm. 9) 185. 192 Abb. 12.

96 Lollini (Anm. 50) 294-299. 304. 309 Abb. 20. 26. 31.

97 Lollini (Anm. 33) 181. 183 Abb. 1. 2.

98 Panutti (Anm. 16) 175-186. 188-202 Abb. 15-30; R. Peroni, Osservazioni sul significato della serie stratigrafica di Grotta a Male, Bullettino di Paletnologia Italiana 78, 1969, 250-251. 257-258.

99 Vigliardi - Ghezzi (Anm. 25) 165 Abb. 16; 167 Abb. 17; 181 Abb. 19; 183 Abb. 20; 186 Abb. 21; 199 Abb. 25; 203 Abb. 26.

${ }^{100}$ Cassano - Cazzella - Manfrechini (Anm. 22) 159-161 Abb. 78-80.

${ }^{101}$ Bréa - Cavalier (Anm. 49) 705-709 Taf. 196-205.

${ }^{102}$ G. Cremonesi, L'eneolitico e l'etá del bronzo nelle alte valli dell'Agri e del Sinni, in: Aa. Vv. (Hrsg.), Attività archeologica in Basilicata 1964-1977. Scritti in onore di Dinu Adamesteanu (1980) 425-426.

${ }^{103}$ G. Gangemi - R. Collina, Casalvelino - Località Torricelli, Apollo 6, 1985-88, 397-398.

${ }^{104}$ G. Patroni, Riparo dello Zachito, Archivo per l'Antropologia e l'Etnologia 32/2, 1903, 197.

${ }^{105}$ P. Carucci, La grotta preistorica di Pertosa (1907) 1-99. 


\section{Fundkatalog}

Im Katalog verwendete Abkürzungen

$$
\begin{aligned}
& \text { Abr. = Abrollungsgrad } \\
& \mathrm{B}=\text { Breite } \\
& \mathrm{BaDm}=\text { Bauchdurchmesser } \\
& \text { BaWst. = Bauchwandstärke } \\
& \text { BdDm = Bodendurchmesser } \\
& \text { BdWst. = Bodenwandstärke } \\
& \mathrm{D}=\text { Dicke }
\end{aligned}
$$

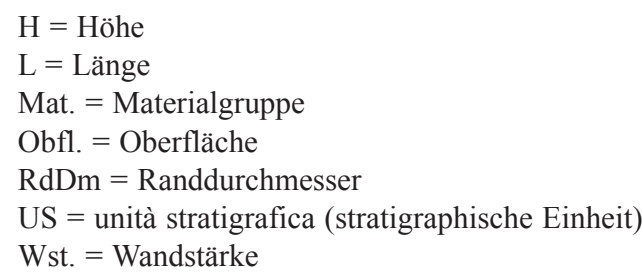

Inv. 180/93-2.1

Schnitt 1/93, US 3

Wandfragment eines nicht näher bestimmbaren Gefäßes Obfl. außen und innen nicht mehr vorhanden; Wst. 7-9 mm; Abr.: abgerundet; Mat.: 3.1; Farbe außen: hellgrau (10YR5/2), Farbe Bruch: hellbraun (10YR3/1), Farbe innen: dunkelgrau (7.5YR6/4).

Inv. 247/93-3.1

Schnitt 2/93, US 3

Wandfragment eines nicht näher bestimmbaren Gefäßes Obfl. außen und innen geglättet; Wst. 9-2.1 mm; Abr.: abgerundet; Mat.: 3.1; Farbe außen: hellgrau (10YR6/3), Farbe Bruch: dunkelgrau (7.5YRN3), Farbe innen: hellbraun (10YR6/4).

\section{Inv. 713/93-3.1 (Taf. 3, 9)}

Schnitt 7/93, US 6

Rand bis Unterteil einer Knickwandschüssel

Obfl. außen und innen geglättet; H $48 \mathrm{~mm}$, B $60 \mathrm{~mm}$, RdDm 25 cm, BaDm 23.5 cm, Wst. 5-9 mm; Abr.: wenig abgerundet; Mat.: 2.3; Farbe außen: beige (10YR7/4), Farbe Bruch: dunkelgrau (10YR3/1), Farbe innen: beige (10YR7/4).

\section{Inv. 203/96-114.1 (Taf. 1, 2)}

Schnitt 2/96, US 3

Oberteil eines Henkelgefäßes mit Trichterhals und gewölbter Schulter

Obfl. außen und innen sehr gut geglättet und poliert; H 66 mm, B 40 mm, Wst. 5-7 mm, Henkel-Wst. 85 mm; Abr.: abgerundet, Mat.: 1; Farbe außen: $\operatorname{rot}(2.5 \mathrm{YR} 4 / 8)$, Farbe Bruch: dunkelgrau (2.5YR3/1), Farbe innen: rot (2.5YR4/8).

Inv. 203/96-115.1 (Taf. 4, 9)

Schnitt 2/96, US 3

Rand einer kreisförmigen Platte

Obfl. oben mit Hand geglättet und unten sehr unregelmäßig; H 28 mm, B 105 mm, RdDm 20 mm, Wst. 20-26 mm; Abr.: gezackt; Mat.: 4.4; Farbe oben: $\operatorname{rot}(2.5 \mathrm{YR} 4$ / 6), Farbe Bruch: dunkelrot (2.5YR3/6), Farbe unten: dunkelrot (2.5YR3/6).

Inv. 203/96-115.2-3

Schnitt 2/96, US 3

Wandfragmente eines nicht näher bestimmbaren GefäBes
Obfl. oben sehr gut geglättet und unten geglättet; Wst. 10 mm; Abr.: abgerundet; Mat.: 3.1; Farbe oben: graubraun (10YR3/2), Farbe Bruch: dunkelgrau (10YR4/1), Farbe unten: braun (10YR4/3).

\section{Inv. 203/96-116.1 (Taf. 3, 7)}

Schnitt 2/96, US 3

Rand bis Unterteil einer stark profilierten Knickwandschüssel

Obfl. außen und innen sehr gut geglättet und poliert, wobei Politur außen praktisch nicht mehr vorhanden; H 32 mm, B 35 mm, RdDm 14 mm, BaDm 15.3 mm, Wst. 35-75 mm; Abr.: abgerundet; Mat.: 2.8; Farbe außen: sehr hellorange (5YR7/8), Farbe Bruch: dunkelbraun (5YR3/ $3)$, Farbe innen: sehr hellorange (5YR7/8).

\section{Inv. 203/96-116.2}

Schnitt 2/96, US 3

Wandfragment eines nicht näher bestimmbaren Gefäßes.

\section{Inv. 203/96-119.1}

Schnitt 2/96, US 3

Unretuschierter Abschlag

L 30 mm, B 20 mm, D 2-5 mm; Querschnitt: trapezoid; Farbe: dunkelbraun (10R3/1).

\section{Inv. 203/96-119.2}

Schnitt 2/96, US 3

Unretuschierter Abschlag

L 25 mm, B 15 mm, D 1-3 mm; Querschnitt: trapezoid; Farbe: rotbraun $(2.5 \mathrm{YR} 4 / 4)$.

\section{Inv. 203/96-119.3}

Schnitt 2/96, US 3

Unretuschierter Abschlag

L 50 mm, B 25 mm, D 3-7 mm; Querschnitt: trapezoid; Farbe: braun $(7.5 \mathrm{YR} 5 / 3)$.

Inv. 211/96-30.1 (Taf. 2, 6)

Schnitt 2/96, US 11

Fragment eines Henkels

Obfl. außen und innen mit der Hand unregelmäßig geglättet; H 47 mm, B 82 mm, Wst. 15-24.5 mm; Abr.: wenig abgerundet; Mat.: 2.3; Farbe außen: rot (2.5YR5/ $8)$, Farbe Bruch: schwarz (2.5YR2/0), Farbe innen: rot (2.5YR5/8). 


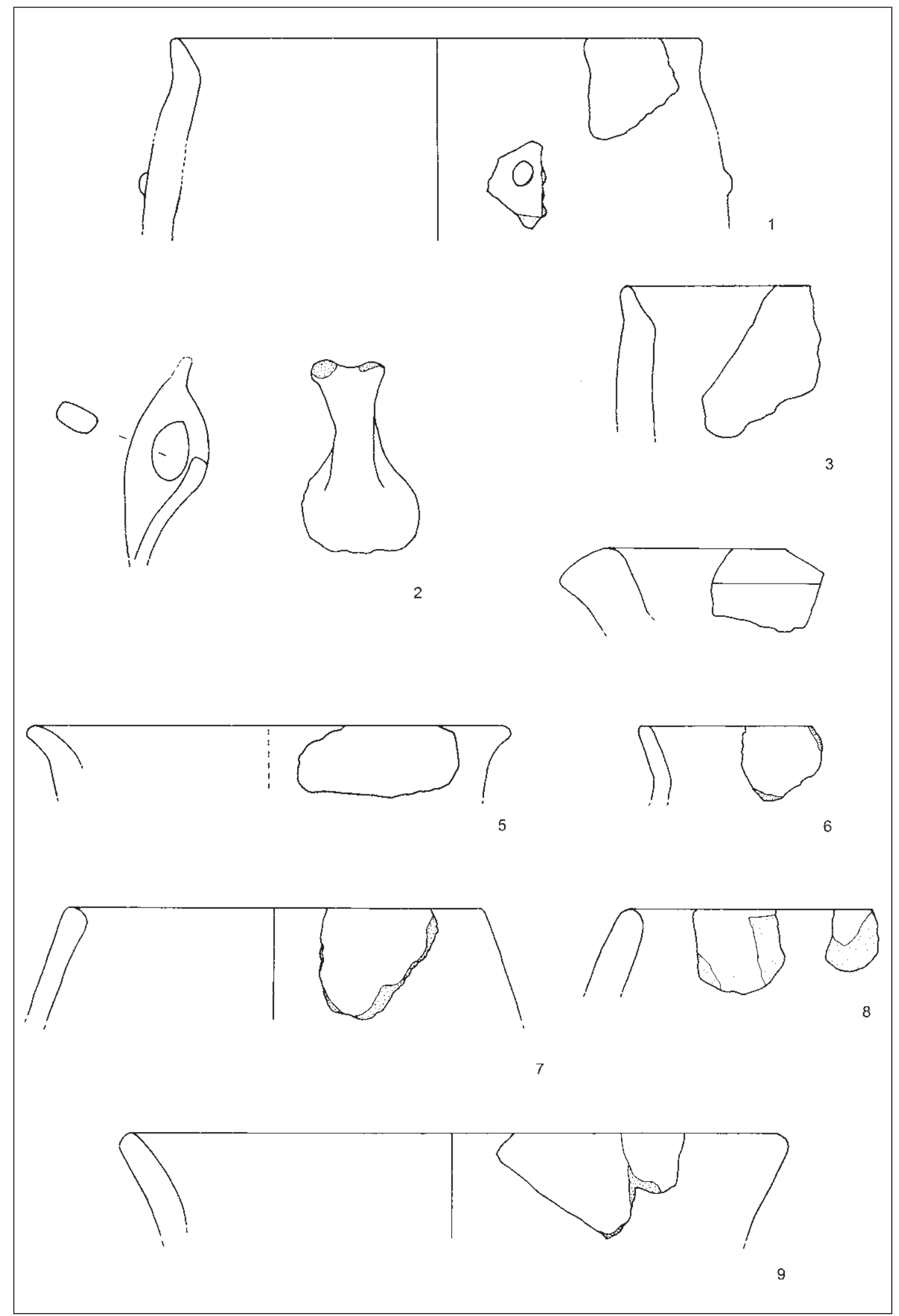

Tafel 1 Bronzezeitliche Topfformen aus Velia. M 1:2 


\section{Inv. 215/96-62.1 (Taf. 4, 5)}

Schnitt 2/96, US 15

Fragment eines Henkels

Obfl. außen und innen nicht mehr vorhanden; H $52 \mathrm{~mm}$, B 59 mm, Wst. 13.5-20 mm; Abr.: abgerundet; Mat.: 3.1; Farbe außen: grau (10YR4/1), Farbe Bruch: dunkelgrau (10YR3/1), Farbe innen: grau (10YR4/1).

Inv. 216/96-25.1 (Taf. 1, 3)

Schnitt 2/96, US 16

Oberteil eines Topfes mit gewölbter Schulter

Obfl. außen und innen glatt mit Spuren langsamer Drehscheibe; H 52 mm, B 40 mm, Wst. 13 mm; Abr.: wenig abgerundet; Mat.: 4.5; Farbe außen: dunkelgelbbraun (7.5YR5/8), Farbe Bruch: braun (10YR4/4), Farbe innen: dunkelgelbbraun (10YR5/6).

Inv. 216/96-26.1-3 (Taf. 2, 8)

Schnitt 2/96, US 16

Unterteil eines Topfes mit gewölbtem Unterteil und flachem Boden

Obfl. außen: handgeglättet, innen: grob, sehr unregelmäßig geglättet; H 60 mm, B 105 mm, BaDm 105 mm, BaWst. 8 mm, BdWst. 13 mm; Abr.: abgerundet; Mat.: 4.5; Farbe außen: rotbraun (5YR5/6), Farbe Bruch: braunrot (5YR4/6), Farbe innen: schwarz (10YR2/1).

\section{Inv. 216/96-27.1, 2, 4, 5}

Schnitt 2/96, US 16

4 Wandfragmente unterschiedlicher, nicht näher bestimmbarer Gefäße.

Inv. 216/96-27.3 (Taf. 3, 8)

Schnitt 2/96, US 16

Wandfragment einer stark profilierten Knickwandschüssel

Obfl. außen und innen geglättet und poliert; H $20 \mathrm{~mm}$, B $34 \mathrm{~mm}$, Wst. 5-7 mm; Abr.: abgerundet; Mat.: 2.5; Farbe außen: orange (5YR6/8), Farbe Bruch: schwarz (7.5YR2/0), Farbe innen: grau (5Y4/1).

\section{Inv. 224/96-14.1}

Schnitt 2/96, US 24

Gewölbter Boden eines nicht näher bestimmbaren GefäBes

Obfl. außen nicht mehr vorhanden und innen geglättet; Wst. $10 \mathrm{~mm}$; Abr.: wenig abgerundet; Mat.: 2.1; Farbe außen: orange (2.5YR/5.8), Farbe Bruch: dunkelgraubraun (10YR3/2), Farbe innen: orange (5YR6/8).

\section{Inv. 224/96-15.1}

Schnitt 2/96, US 24

Gewölbte Wand eines nicht näher bestimmbaren Gefäßes

Obfl. außen und innen glatt mit Spuren langsamer Drehscheibe; Wst. 8 mm; Abr.: abgerundet; Mat.: 4.5; Farbe außen: orange (5YR6/8), Farbe Bruch: braun (7.5YR5/4), Farbe innen: orange (5YR6/8)
Inv. 225/96-13.1 (Taf. 4, 6)

Schnitt 2/96, US 25

Fragment eines doppelten zylindrischen Henkels

Obfl. außen und innen nicht mehr vorhanden; H $55 \mathrm{~mm}$, B 83 mm, Wst. 17.5-25 mm; Abr.: abgerundet; Mat.: 2.8; Farbe außen: orange (5YR6/8), Farbe Bruch: dunkelgrau (5YR3/1), Farbe innen: orange (5YR6/8).

Inv. 225/96-14.1 (Taf. 4, 10)

Schnitt 2/96, US 25

Rand einer kreisförmigen Platte

Obfl. oben handgeglättet (mit Fingerabdrücken) und unten grob modelliert; H $28 \mathrm{~mm}$, B $89 \mathrm{~mm}$, RdDm $30 \mathrm{~mm}$, Wst. $22 \mathrm{~mm}$; Mat.: 5; Abr.: abgerundet; Farbe außen: braun (7.5YR5/4), Farbe Bruch: braun (10YR5/3), Farbe innen: braun (7.5YR5/4).

\section{Inv. 225/96-15.1}

Schnitt 2/96, US 25;

Rand bis Unterteil einer Schüssel mit weichem S-Profil und leicht ausladendem Rand

Obfl. außen und innen nicht mehr vorhanden; Wst. $5 \mathrm{~mm}$; Abr.: abgerundet; Mat.: 3.3; Farbe außen: hellbraungrau (10YR6/2), Farbe Bruch: grau (10YR4/1), Farbe innen: hellbraungrau (10YR6/2).

\section{Inv. 225/96-15.2-11}

Schnitt 2/96, US 25

10 Wandfragmente unterschiedlicher, nicht näher bestimmbarer Gefäße.

Inv. 226/96-41.1 (Taf. 1, 7)

Schnitt 2/96, US 26

Eingezogenes Rand- bzw. Schulterfragment eines Topfes Obfl. außen geglättet und innen geglättet, teilweise hoch poliert; H 38 mm, B 41 mm, RdDm $14 \mathrm{~mm}$, Wst. $7.5 \mathrm{~mm}$; Abr.: wenig gezackt; Mat.: 4.5; Farbe außen: hellrot (2.5YR6/8), Farbe Bruch: dunkelgrau (2.5YR3/1), Farbe innen: dunkelbraun (10YR3/2).

Inv. 226/96-41.2 (Taf. 1, 6)

Schnitt 2/96, US 26

Rand bis Schulter eines Topfes mit kurzem ausladendem Hals und gerader Schulter

Obfl. außen und innen glatt mit Spuren langsamer Drehscheibe; H 25 mm, B 27 mm, Wst. 6 mm; Abr.: gezackt; Mat.: 2.5; Farbe außen: rotbraun (5YR5/6), Farbe Bruch: dunkelgrau (5YR3/1), Farbe innen: dunkelgrau (5YR3/1).

Inv. 226/96-41.3 (Taf. 1, 4)

Schnitt 2/96, US 26

Stark ausladender Rand eines Topfes

Obfl. außen und innen nicht mehr vorhanden; H $28 \mathrm{~mm}$, B $38 \mathrm{~mm}$, Wst. 18.5-22 mm; Abr.: wenig abgerundet; Mat.: 2.3; Farbe außen: hellorange (2.5YR6/6), Farbe Bruch: grau (2.5YR5/1), Farbe innen: hellorange (2.5YR6/6).

Inv. 226/96-42.1 (Taf. 2, 7)

Schnitt 2/96, US 26

Gewölbte Schulter eines Topfes 


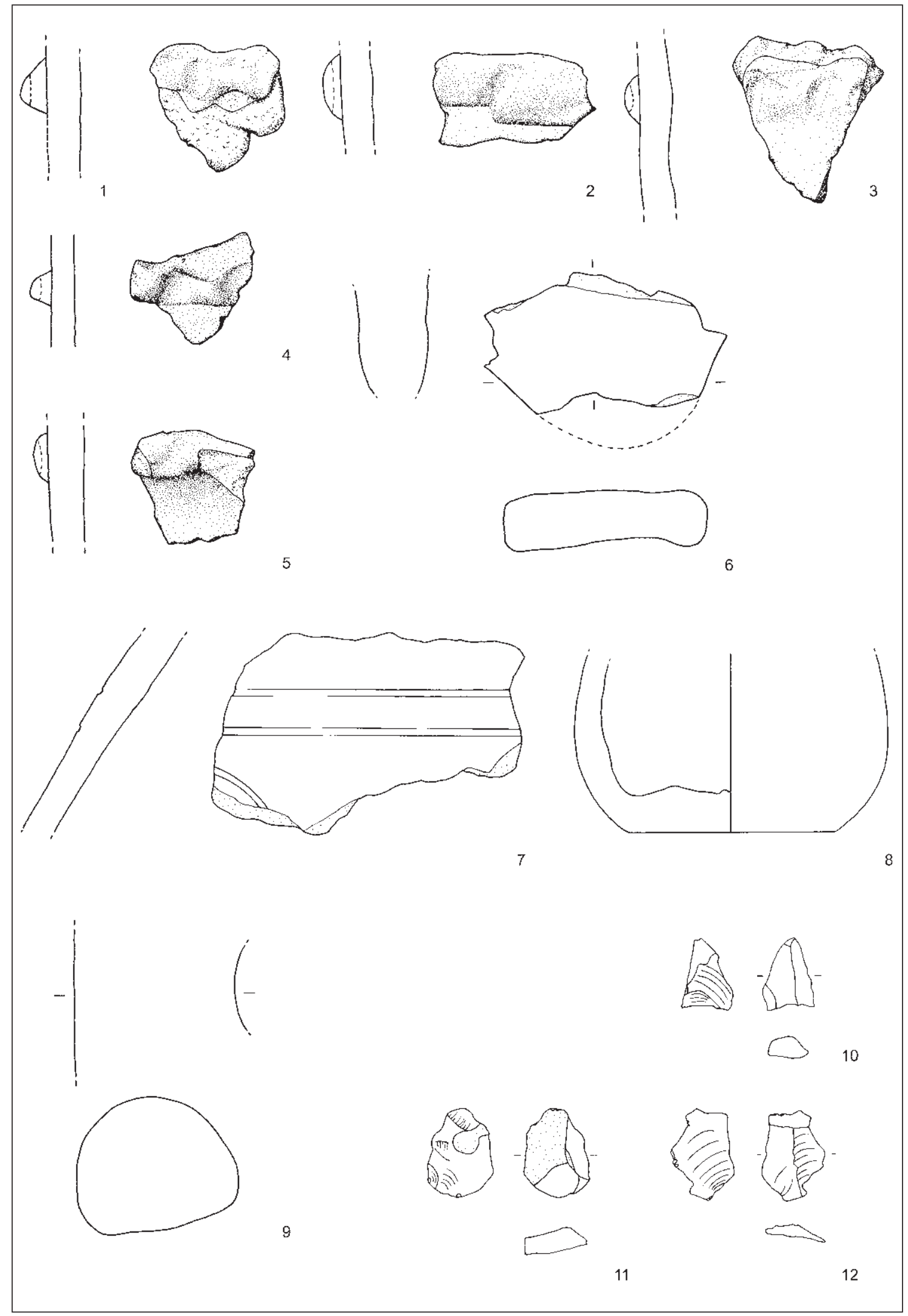

Tafel 2 Bronzezeitliche Topf- und Sonderformen sowie Silices aus Velia. M 1:2 
Obfl. außen und innen geglättet; H 69 mm, B 105 mm, Wst. 10-13 mm; Abr.: wenig gezackt; Mat.: 2.7; Farbe außen: braun (7.5YR5/4), Farbe Bruch: dunkelgelbbraun (7.5YR4/6), Farbe innen: hellbraun (7.5YR6/4); am oberen Schulterteil zwei eingedrückte horizontale parallele Linien, darunter eine ebenfalls eingedrückte kreis- oder halbkreisförmige Linie.

Inv. 226/96-43.1 (Taf. 4, 7)

Schnitt 2/96, US 26

Wandfragment eines Henkelgefäßes

Verzierung außen: oberhalb des Bauchumbruches horizontal gelegte (Finger-)Tupfenleiste.

Obfl. außen unregelmäßig geglättet und innen nicht mehr vorhanden; H $61 \mathrm{~mm}$, B $84 \mathrm{~mm}$, Wst. 6-9 mm; Abr.: wenig abgerundet; Mat.: 4.5; Farbe außen: hellbraun (7.5YR6/4), Farbe Bruch: schwarz (7.5YR2/0), Farbe innen: hellbraun (7.5YR6/4).

Inv. 226/96-44.1 (Taf. 3, 10)

Schnitt 2/96, US 26

Rand einer großen Schüssel

Obfl. außen grob geglättet und innen geglättet; H 90 mm, B 103 mm, RdDm 50 mm, Wst. 19-40 mm; Abr.: gezackt; Mat.: 4.5; Farbe außen: hellorange (5YR7/6), Farbe Bruch: hellorange (5YR7/6), Farbe innen: hellorange (5YR7/6).

Inv. 226/96-44.2 (Taf. 2, 9)

Schnitt 2/96, US 26

Fragment eines unbestimmbaren Gegenstandes Obfl. außen unregelmäßig modelliert; H 50 mm, B 57 mm, Wst. 45-56 mm; Abr.: gezackt; Mat.: 4.5; Farbe außen: braunorange (5YR6/6), Farbe Bruch: grau (5YR5/1).

Inv. 226/96-44.3-84

Schnitt 2/96, US 26

82 Wandfragmente unterschiedlicher, nicht näher bestimmbarer Gefäße.

\section{Inv. 227/96-26.1}

Schnitt 2/96, US 27

Rand einer kreisförmigen Platte

Obfl. oben geglättet und unten sehr unregelmäßig; Wst. 20-25 mm; Abr.: wenig gezackt; Mat.: 4.4; Farbe oben: rot (2.5YR4/6), Farbe Bruch: dunkelrot (2.5YR3/6), Farbe unten: dunkelrot (2.5YR3/6).

Inv. 227/96-27.1 (Taf. 4, 8)

Schnitt 2/96, US 27

Wandfragment eines Topfes; Verzierung außen: horizontal gelegte Leiste mit Fingerabdrücken.

Obfl. außen und innen nicht mehr vorhanden; H $63 \mathrm{~mm}$, B $80 \mathrm{~mm}$, Wst. 12-13 mm; Abr.: abgerundet Mat.: 2.2; Farbe außen: braungelb (7.5YR7/6), Farbe Bruch: dunkelbraun (10YR3/3), Farbe innen: braungelb (7.5YR7/6).

Inv. 227/96-27.2-7

Schnitt 2/96, US 27

6 Wandfragmente unterschiedlicher, nicht näher bestimmbarer Gefäße.
Inv. 227/96-27.8 (Taf. 3, 1)

Schnitt 2/96, US 27

Ausladender Rand einer Schale mit gewölbter Wand Obfl. außen und innen glatt mit Spuren langsamer Drehscheibe; H 25 mm, B 22 mm, Wst. 7 mm; Abr.: gezackt; Mat.: 3.1; Farbe außen: dunkelbraun (2.5YR2/4), Farbe Bruch: dunkelgrau (7.5YR4.1), Farbe innen: dunkelbraun (2.5YR2/4).

Inv. 227/96-27.9-10 (Taf. 1, 1)

(gehört zu Inv. 257/96-30.2, 2007/97-7.1-3 und 2025/974.2)

Schnitt 2/96, US 27

Schulter eines Topfes; Handhaben: auf der Schulter runde Knubbe

Obfl. außen und innen glatt mit Spuren langsamer Drehscheibe; H 30 mm, B 20 mm, Wst. 12 mm; Abr.: gezackt; Mat.: 4.2; Farbe außen: dunkelbraun (2.5YR2/4), Farbe Bruch: hellbraun (5YR6/6), Farbe innen: dunkelrot (2.5YR3/6).

\section{Inv. 227/96-27.11-14}

Schnitt 2/96, US 27

4 Wandfragmente unterschiedlicher, nicht näher bestimmbarer Gefäße.

Inv. 228/96-1.1 (Taf. 2, 12)

Schnitt 2/96, US 28

Unretuschierter Abschlag, proximal erhalten

L $31 \mathrm{~mm}$, B 24 mm, D 1-4 mm; Querschnitt: trapezoid; Farbe: dunkelbraun (10R3/1).

Inv. 228/96-1.2 (Taf. 2, 11)

Schnitt 2/96, US 28

Unretuschierter Abschlag mit Cortexresten in beiden Seiten, ventral: unregelmäßige flächige Retuschen

L $30 \mathrm{~mm}$, B $22 \mathrm{~mm}$, D 2-8 mm; Querschnitt: trapezoid; Farbe: braun (7.5YR5/3).

Inv. 228/96-2.1 (Taf. 3, 4)

Schnitt 2/96, US 28

Ausladender Rand bis Unterteil einer weichen profilierten Schüssel

Obfl. außen und innen sehr gut geglättet und z. T. Politur noch vorhanden; H $43 \mathrm{~mm}$, B $57 \mathrm{~mm}$, RdDm 8 mm, Wst. 5-8 mm; Abr.: wenig abgerundet; Mat.: 2.2; Farbe außen: dunkelgrau (10YR3/1), Farbe Bruch: dunkelgrau (10YR3/1), Farbe innen: dunkelgrau (10YR3/1).

Inv. 228/96-2.2-3 (Taf. 1, 8)

Schnitt 2/96, US 28

Eingezogener Rand und Schulter eines Topfes

Obfl. außen und innen geglättet; H 29 mm, B 49 mm, Wst. $12 \mathrm{~mm}$; Abr.: gezackt; Mat.: 2.1; Farbe außen: braungelb (7.5YR7/6), Farbe Bruch: gelbbraun (7.5YR6/ $6)$, Farbe innen: braungelb (7.5YR7/6).

\section{Inv. 228/96-2.4}

Schnitt 2/96, US 28

Konischer Unterteil einer Schüssel mit flachem Boden Obfl. außen und innen geglättet; Wst. $8.5 \mathrm{~mm}$; Abr.: 


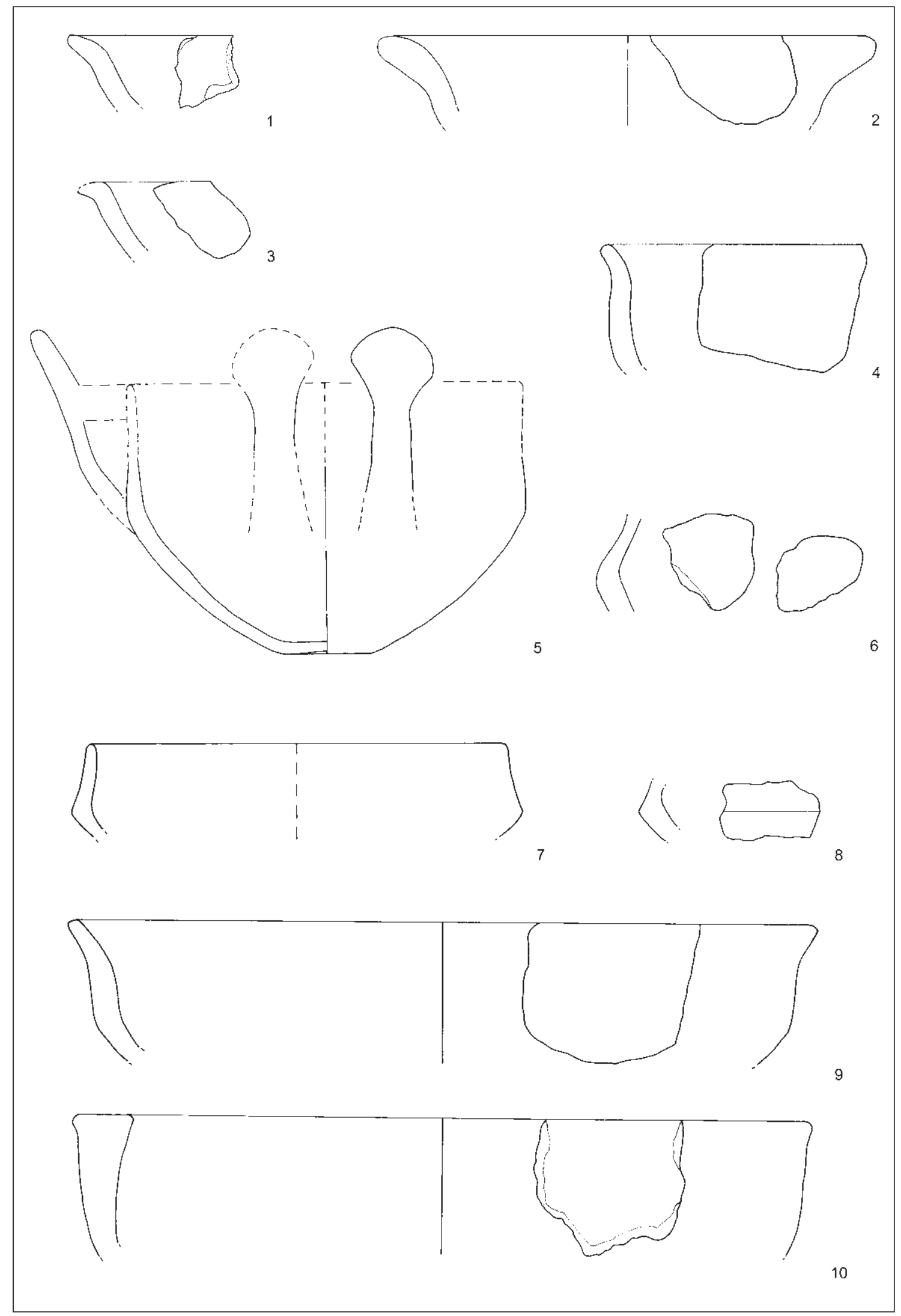

Tafel 3 Bronzezeitliche Schüsselformen aus Velia. M 1:2 
wenig abgerundet; Mat:: 2.6; Farbe außen: gelbbraun (7.5YR6/6), Farbe Bruch: schwarz (5YR2.5/1), Farbe innen: braungelb (7.5YR7/6)

Inv. 228/96-3.1-11 (Taf. 3, 5)

(gehört zu Inv. 2000/97-1.1-4, 2000/97-2.1)

Schnitt 2/96, US 28

Oberteil bis Boden einer Knickwandschüssel mit leicht eingedelltem Boden

Obfl. außen und innen sehr gut geglättet und poliert; H 67 mm, B 68 mm, BdDm 6 mm, BaDm 13.6 mm, Wst. 4-6.5 mm; Abr.: wenig abgerundet; Mat.: 2.1; Farbe außen: schwarz (5YR2/1), Farbe Bruch: grau (5YR5/1), Farbe innen: schwarz (5YR2/1).

Inv. 228/96-3.12 (Taf. 4, 2)

Schnitt 2/96, US 28

Boden einer Schüssel(?) mit tiefem Omphalos

Obfl. außen und innen geglättet; H $14 \mathrm{~mm}$, B $58 \mathrm{~mm}$, BdDm 4 mm, Wst. 6-9 mm; Abr.: wenig gezackt; Mat.: 4.5; Farbe außen: grau (7.5YR4/1), Farbe Bruch: dunkelgrau (7.5YR3/1), Farbe innen: grau (7.5YR4/1).

\section{Inv. 228/96-4.1-4}

(gehört wahrscheinlich zu Inv. 2028/97-3.3-4)

Schnitt 2/96, US 28

4 Wandfragmente eines nicht näher bestimmbaren GefäBes

Obfl. außen und innen geglättet; Wst. $12 \mathrm{~mm}$; Abr.: wenig gezackt; Mat.: 2.2; Farbe außen: hellbraun (5YR6/6), Farbe Bruch: braunrot (5YR4/6), Farbe innen: braungelb (7.5YR7/6).

\section{Inv. 228/96-4.5-6}

Schnitt 2/96, US 28

2 Wandfragmente eines nicht näher bestimmbaren GefäBes

Obfl. außen und innen geglättet; Wst. 5 mm; Abr:: abgerundet; Mat.: 4.5; Farbe außen: braungelb (7.5YR7/6), Farbe Bruch: orange (5YR6/8), Farbe innen: braungelb (7.5YR7/6).

\section{Inv. 228/96-4.7-10}

(gehört wahrscheinlich zu Inv. 2059/97-3.1)

Schnitt 2/96, US 28

Wandfragmente eines nicht näher bestimmbaren GefäBes

Obfl. außen und innen geglättet; Wst. $10 \mathrm{~mm}$; Abr.: wenig abgerundet; Mat.: 2.7; Farbe außen: beige (7.5YR7/ 4), Farbe Bruch: braun (10YR4/4), Farbe innen: beige (7.5YR7/4).

Inv. 228/96-4.11-12 (Taf. 3, 6)

Schnitt 2/96, US 28

Ober- bis Unterteil einer stark profilierten Knickwandschüssel

Obfl. außen und innen geglättet; H $33 \mathrm{~mm}$, B $60 \mathrm{~mm}$, Wst. 5-8 mm; Abr.: wenig gezackt; Mat.: 2.4; Farbe außen: rot (2.5YR5/6), Farbe Bruch: grau (10YR.5/1), Farbe innen: rot $(2.5 \mathrm{YR} 5 / 6)$.

\section{Inv. 228/96-4.13-14}

Schnitt 2/96, US 28

Wandfragmente eines nicht näher bestimmbaren Gefäßes

Obfl. außen grob modelliert und innen geglättet; Wst. 13 mm; Abr.: gezackt; Mat.: 3.1; Farbe außen: grau (7.5YR5/1), Farbe Bruch: schwarz (7.5YR2.0), Farbe innen: grau (7.5YR5/1).

\section{Inv. 228/96-4.15-57}

Schnitt 2/96, US 28; 42

Wandfragmente unterschiedlicher, nicht näher bestimmbarer Gefäße.

\section{Inv. 228/96-5.1-2}

Schnitt 2/96, US 28

Fragment einer Platte

Obfl. oben mit Hand geglättet und unten sehr unregelmäßig; Wst. 20-25 mm; Abr.: gezackt; Mat.: 4.4; Farbe oben: rot (2.5YR4/6), Farbe Bruch: dunkelrot (2.5YR3/ $6)$, Farbe unten: dunkelrot (2.5YR3/6).

\section{Inv. K 8/96-1.1-2}

Schnitt 2/96, US 28, Nordostprofil

Wandfragmente eines nicht näher bestimmbaren Gefäßes.

\section{K 9/96-1.1}

Schnitt 2/96, US 28, Nordostprofil

Wandfragment eines nicht näher bestimmbaren Gefäßes.

\section{K 10/96-1.1}

Schnitt 2/96, US 28, Nordostprofil

Wandfragment eines nicht näher bestimmbaren GefäBes.

Inv. 229/96-68.1-2 (Taf. 4, 3)

Schnitt 2/96, US 29

Konischer Unterteil einer Schüssel mit leicht eingedelltem Boden

Obfl. außen und innen geglättet; H $21 \mathrm{~mm}$, B $99 \mathrm{~mm}$, BdDm $6 \mathrm{~mm}$, Wst. 8-10 mm; Abr.: wenig abgerundet; Mat.: 2.8; Farbe außen: grau (10YR4/1), Farbe Bruch: braun (10YR5/3), Farbe innen: dunkelgrau (10YR3/1).

\section{Inv. 229/96-69.1}

\section{Schnitt 2/96, US 29}

Flacher Boden eines Gefäßes

Obfl. außen und innen geglättet; Wst. $12 \mathrm{~mm}$; Abr:: gezackt; Mat.: 3.1; Farbe außen: hellbraun (7.5YR6/4), Farbe Bruch: braun (10YR5/3), Farbe innen: hellbraun (7.5YR6/4).

Inv. 229/96-70.1-3 (Taf. 2, 1)

Schnitt 2/96, US 29

Wandfragmente eines Topfes

Verzierung außen: horizontal gelegte Leiste mit Fingerabdrücken.

Obfl. außen und innen geglättet; H $43 \mathrm{~mm}$, B $46 \mathrm{~mm}$, Wst. $12 \mathrm{~mm}$; Abr.: wenig abgerundet; Mat.: 3.2; Farbe 


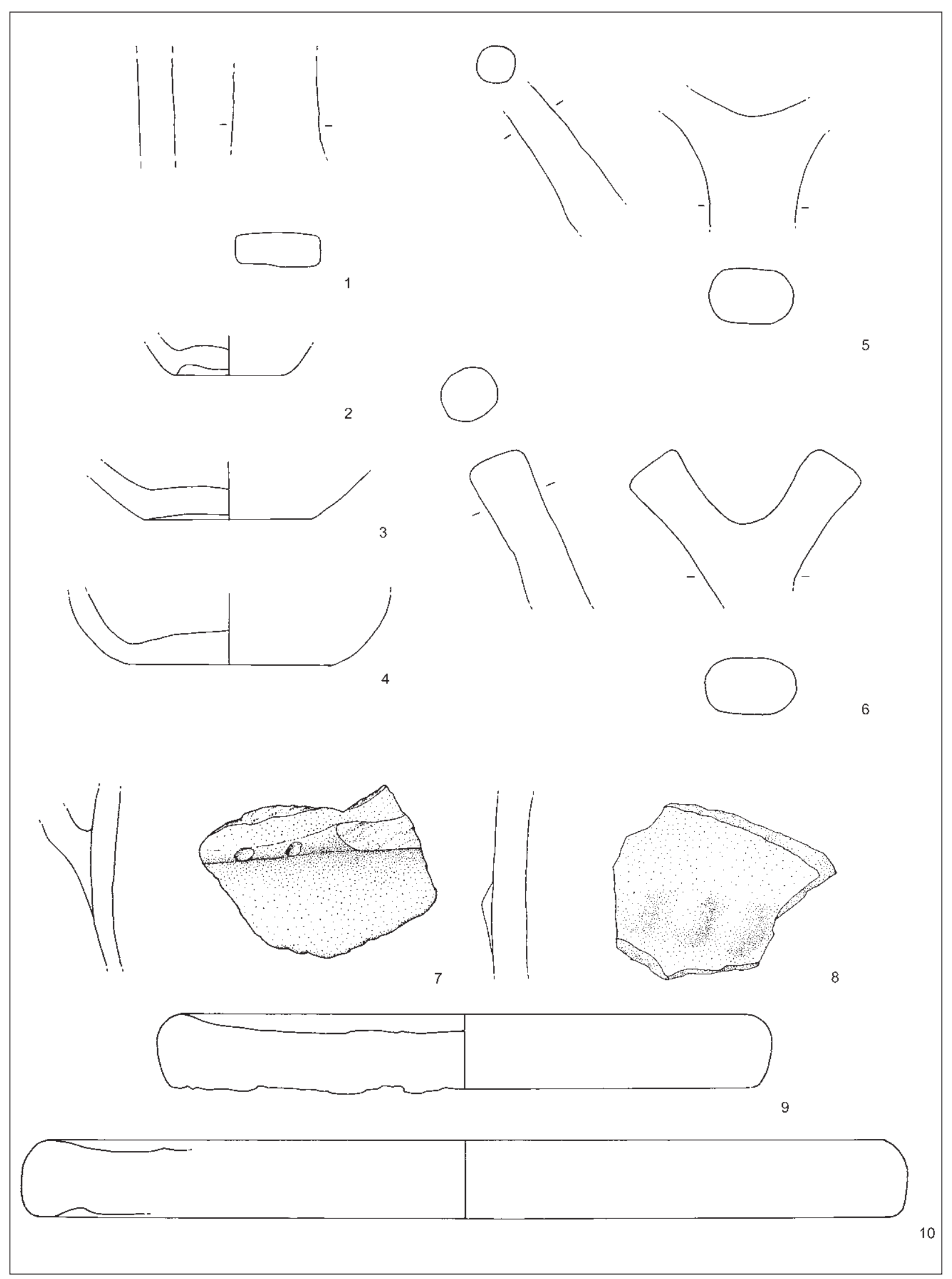

Tafel 4 Bronzezeitliche Topf- und Schüsselformen sowie Keramikplatten aus Velia. M 1:2 
außen: orange (2.5YR6/8), Farbe Bruch: rot (2.5YR4/8), Farbe innen: orange (2.5YR6/8).

\section{Inv. 229/96-70.4}

Schnitt 2/96, US 29

Wandfragment eines nicht näher bestimmbaren Gefäßes.

Inv. 229/96-70.5 (Taf. 4, 1)

Schnitt 2/96, US 29

Fragment eines Bandhenkel

Obfl. außen und innen nicht mehr vorhanden; H $35 \mathrm{~mm}$, B $32 \mathrm{~mm}$, Wst. $12 \mathrm{~mm}$; Abr.: wenig abgerundet; Mat.: 2.8; Farbe außen: schwarz (7.5YR2/0), Farbe Bruch: schwarz (7.5YR2/0), Farbe innen: schwarz (7.5YR2/0).

\section{Inv. 229/96-70.6-33}

Schnitt 2/96, US 29; 28

Wandfragmente unterschiedlicher, nicht näher bestimmbarer Gefäße.

Inv. 229/96-71.1 (Taf. 2, 10)

Schnitt 2/96, US 29

Unretuschierter Abschlag

L 24 mm, B 18 mm, D 1-7 mm; Querschnitt: trapezoid; Farbe: braun $(7.5 \mathrm{YR} 5 / 3)$.

\section{Inv. 230/96-29.1-4}

Schnitt 2/96, US 30

Wandfragmente eines nicht näher bestimmbaren GefäBes

Obfl. außen und innen geglättet; Wst. $12.5 \mathrm{~mm}$; Abr.: wenig abgerundet; Mat.: 3.1; Farbe außen: braungelb (7.5YR7/6), Farbe Bruch: grau (7.5YR4/1), Farbe innen: braungelb (7.5YR7/6).

\section{Inv. 253/96-14.1-5}

Schnitt 2/96, US 3

Gewölbtes Wandfragment eines nicht näher bestimmbaren Gefäßes

Obfl. außen und innen glatt mit Spuren langsamer Drehscheibe; Wst. 10.5 mm; Abr.: gezackt; Mat.: 3.1; Farbe außen: schwarz (2.5YR2/0), Farbe Bruch: rot (2.5YR4/ 8), Farbe innen: dunkelbraun (2.5YR2/4).

Inv. 257/96-30.1 (Taf. 3, 3)

Schnitt 2/96, US 27 und 29

Rand bis Unterteil einer Schale mit gewölbter Wand Obfl. außen und innen glatt mit Spuren langsamer Drehscheibe; H 26 mm, B 33 mm, Wst. 7 mm; Abr.: gezackt; Mat.: 3.1; Farbe außen: dunkelrotbraun (2.5YR3/4), Farbe Bruch: schwarz (2.5YR2/5), Farbe innen: schwarz (2.5YR2/5).

Inv. 257/96-30.2 (Taf. 1, 1)

(gehört zu Inv. 227/96-27.9-10, 2007/97-7.1-3 und 2025/ 97-4.2)

Schnitt 2/96, US 27 und 29

Rand bis Schulter eines Topfes mit nach außen geknicktem Rand und gewölbter Schulter

Obfl. außen und innen glatt mit Spuren langsamer Dreh- scheibe; H 35 mm, B 32 mm, RdDm 18 mm, Wst. 11 mm; Abr.: gezackt; Mat.: 4.1; Farbe außen: dunkelrotbraun (2.5YR3/4), Farbe Bruch: dunkelbraun (2.5YR3/ 2), Farbe innen: rot (2.5YR4/6).

Inv. 257/96-30.3-4 (Taf. 4, 4)

Schnitt 2/96, US 27 und 29

Gewölbter Unterteil einer Schüssel mit flachem Boden Obfl. außen und innen sehr gut geglättet; H $25 \mathrm{~mm}$, B $115 \mathrm{~mm}$, BdDm $8 \mathrm{~mm}$, Wst. 8-12 mm; Abr:: wenig abgerundet; Mat.: 3.2; Farbe außen: hellbraun (5YR6/6), Farbe Bruch: dunkelgrau (2.5YR3/1), Farbe innen: hellorange (5YR7/6).

\section{Inv. 257/96-30.5-23}

Schnitt 2/96, US 27 und 29; 19

Wandfragmente unterschiedlicher, nicht näher bestimmbarer Gefäße.

\section{Inv. 258/96-5.1}

Schnitt 2/96, US 18

Wandfragment eines nicht näher bestimmbaren Gefäßes Obfl. außen und innen geglättet; Wst. $17 \mathrm{~mm}$; Abr.: wenig abgerundet; Mat.: 3.1; Farbe außen: $\operatorname{rot}(2.5$ YR5/6), Farbe Bruch: dunkelrotbraun (2.5YR3/3), Farbe innen: $\operatorname{rot}(2.5 \mathrm{YR} 5 / 6)$.

\section{Inv. 259/96-4.1}

Schnitt 2/96, US 7

Flacher Boden eines Gefäßes

Obfl. außen und innen geglättet; Wst. $10 \mathrm{~mm}$; Abr.: abgerundet; Mat.: 2.2; Farbe außen: hellbraun (7.5YR6/3), Farbe Bruch: grau (7.5YR5/1), Farbe innen: hellbraun (7.5YR6/3).

\section{Inv. 259/96-4.2}

Schnitt 2/96, US 7

Wandfragment eines nicht näher bestimmbaren Gefäßes Wst. $10 \mathrm{~mm}$.

\section{Inv. 259/96-4.3-4}

Schnitt 2/96, US 7

Wandfragmente eines nicht näher bestimmbaren Gefäßes

Obfl. außen und innen glatt mit Spuren langsamer Drehscheibe; Wst. 9 mm; Abr.: wenig abgerundet; Mat.: 2.7; Farbe außen: rot (2.5YR5/6), Farbe Bruch: dunkelrotbraun (2.5YR3/4), Farbe innen: rot (2.5YR5/6).

Inv. 261/96-1.1-2 (Taf. 1, 9)

Schnitt 2/96, US 26

Ausladender Rand eines Topfes

Obfl. außen und innen geglättet und z. T. noch poliert; H 36 mm, B 64 mm, RdDm 22 mm, Wst. 10 mm; Abr.: wenig abgerundet; Mat.: 3.3; Farbe außen: dunkelorange (5YR5/8), Farbe Bruch: grau (7.5YR5/1), Farbe innen: gelbbraun (7.5YR6/6).

Inv. 261/96-1.3 (Taf. 2, 2)

Schnitt 2/96, US 26

Wandfragmente eines Topfes 
Verzierung außen: horizontal gelegte Leiste mit Fingerabdrücken.

Obfl. außen geglättet und innen nicht mehr vorhanden; H $32 \mathrm{~mm}$, B $56 \mathrm{~mm}$, Wst. $11 \mathrm{~mm}$; Abr.: wenig abgerundet; Mat.: 3.3; Farbe außen: dunkelgelbbraun (7.5YR5/ 8), Farbe Bruch: grau (7.5YR4/1), Farbe innen: dunkelgelbbraun (7.5YR5/8).

\section{Inv. 261/96-2.1-2}

Schnitt 2/96, US 26

Wandfragmente eines nicht näher bestimmbaren Gefäßes

Obfl. außen und innen nicht mehr vorhanden; Wst. 110 mm; Abr.: wenig abgerundet; Mat.: 4.3; Farbe außen: hellrot (2.5YR6/8), Farbe Bruch: hellrot (2.5YR6/8), Farbe innen: hellrot (2.5YR6/8).

\section{Inv. 261/96-2.3}

Schnitt 2/96, US 26

Wandfragmente eines nicht näher bestimmbaren GefäBes

Obfl. außen und innen geglättet; Wst. 11 mm; Abr.: wenig gezackt; Mat.: 4.3; Farbe außen: grau (7.5YR5/1), Farbe Bruch: gelbbraun (7.5YR6/8), Farbe innen: gelbbraun (7.5YR6/8).

Inv. 2000/97-1.1-4 (Taf. 3, 5)

(gehört zu Inv. 228/96-3.1-11, 2000/97-2.1)

Schnitt 2/97, US 28

Henkelfragment einer Knickwandschüssel

Obfl. außen und innen geglättet und poliert; H $56 \mathrm{~mm}$, B $28 \mathrm{~mm}$, Wst. 4-7 mm; Abr.: abgerundet; Mat.: 2.1; Farbe außen: schwarz (5YR2/1), Farbe Bruch: grau (5YR5/1), Farbe innen: schwarz (5YR2/1).

Inv. 2000/97-2.1 (Taf. 3, 5)

(gehört zu Inv. 228/96-3.1-11, 2000/97-1.1-4)

Schnitt 2/97, US 28

Henkelfragment einer Knickwandschüssel

Obfl. außen und innen geglättet und poliert; H $27 \mathrm{~mm}$, B 18 mm, Wst. 4 mm; Abr.: abgerundet; Mat.: 2.1; Farbe außen: schwarz (5YR2/1), Farbe Bruch: grau (5YR5/1), Farbe innen: schwarz (5YR2/1).

Inv. 2007/97-7.1-3 (Taf. 1, 1)

(gehört zu Inv. 227/96-27.9-10, 257/96-30.2, 2007/977.1-3 und 2025/97-4.2)

Schnitt 2/97, US 7

Rand bis Schulter eines Topfes mit nach außen geknicktem Rand und gewölbter Schulter

Obfl. außen und innen glatt mit Spuren langsamer Drehscheibe; Wst. 10 mm; Abr.: gezackt; Mat.: 4.5; Farbe außen: dunkelrotbraun (2.5YR3/4), Farbe Bruch: dunkelbraun $(2.5 \mathrm{YR} 3 / 2)$, Farbe innen: $\operatorname{rot}(2.5 \mathrm{YR} 4 / 6)$.

Inv. 2007/97-7.4-8

Schnitt 2/97, US 7

5 Wandfragmente unterschiedlicher, nicht näher bestimmbarer Gefäße.
Inv. 2025/97-4.1 (Taf. 2, 5)

Schnitt 2/97, US 25

Wandfragment eines Topfes

Verzierung außen: horizontal gelegte Leiste mit Fingerabdrücken.

Obfl. außen und innen nicht mehr vorhanden; H 43 mm, B $42 \mathrm{~mm}$, Wst. $12 \mathrm{~mm}$; Abr.: abgerundet; Mat.: 2.4; Farbe außen: orange (2.5YR6/8), Farbe Bruch: sehr dunkelgelbbraun (10YR4/6), Farbe innen: orange (2.5/6/8).

Inv. 2025/97-4.2 (Taf. 1, 1)

(gehört zu Inv. 227/96-27.9-10, 257/96-30.2 und 2007/ 97-7.1-3)

Schnitt 2/97, US 25

Rand bis Schulter eines Topfes mit nach außen geknicktem Rand und gewölbter Schulter

Obfl. außen und innen geglättet mit Spuren langsamer Drehscheibe; Wst. 14.5 mm; Abr.: gezackt; Mat.: 4.1; Farbe außen: dunkelrotbraun (2.5YR3/4), Farbe Bruch: dunkelbraun (2.5YR3/2), Farbe innen: $\operatorname{rot}(2.5 \mathrm{YR} 4 / 6)$.

Inv. 2028/97-1.1 (Taf. 1, 5)

Schnitt 2/97, US 28

Ausladender Rand eines Topfes

Obfl. außen und innen sehr gut geglättet und hoch poliert; H 25 mm, B 55 mm, RdDm 16 mm, Wst. 5-11 mm; Abr.: gezackt; Mat.: 3.1; Farbe außen: schwarz (7.5YR2/0), Farbe Bruch: dunkelbraun (7.5YR4/4), Farbe innen: schwarz (7.5YR2/0).

Inv. 2028/97-1.2 (Taf. 3, 2)

Schnitt 2/97, US 28

Ausladender Rand einer Schüssel

Obfl. außen sehr gut geglättet und hoch poliert und innen nicht mehr vorhanden; H $30 \mathrm{~mm}$, B $50 \mathrm{~mm}$, RdDm $16 \mathrm{~mm}$, Wst. 8-11 mm; Abr.: gezackt; Mat.: 3.1; Farbe außen: gelbbraun (7.5YR6/6), Farbe Bruch: dunkelbraun (7.5YR3/2), Farbe innen: gelbbraun (7.5YR6/6).

Inv. 2028/97-2.1 (Taf. 2, 3)

Schnitt 2/97, US 28

Wandfragment eines Topfes

Verzierung außen: horizontal gelegte Leiste mit Fingerabdrücken.

Obfl. außen und innen geglättet; H $57 \mathrm{~mm}$, B $47 \mathrm{~mm}$, Wst. 9.5-11 mm; Abr.: wenig gezackt; Mat.: 3.3; Farbe außen: sehr hellorange (5YR7/8), Farbe Bruch: braun (5YR4/2), Farbe innen: dunkelgrau (5YR4/1).

Inv. 2028/97-2.2-3 (Taf. 2, 4)

Schnitt 2/97, US 28

Wandfragmente eines Topfes

Verzierung außen: horizontal gelegte Leiste mit Fingerabdrücken.

Obfl. außen und innen nicht mehr vorhanden; H $38 \mathrm{~mm}$, B $84 \mathrm{~mm}$, Wst. 7-9 mm; Abr.: wenig abgerundet; Mat.: 3.4; Farbe außen: braungelb (7.5YR7/6), Farbe Bruch: dunkelgrau (7.5YR3/1), Farbe innen: gelbbraun (7.5YR6/ $6)$. 


\section{Inv. 2028/97-2.4-5}

Schnitt 2/97, US 28

Wandfragmente eines Topfes

Verzierung außen: horizontale gelegte Leiste mit Fingerabdrücken?

Obfl. außen und innen nicht mehr vorhanden; Wst. $140 \mathrm{~mm}$; Abr.: wenig abgerundet; Mat.: 3.4; Farbe außen: sehr hellrot (2.5YR7/8), Farbe Bruch: rot (2.5YR5/6), Farbe innen: sehr hellrot (2.5YR7/8).

\section{Inv. 2028/97-3.1-2}

Schnitt 2/97, US 28

Wandfragmente eines nicht näher bestimmbaren GefäBes

Obfl. außen und innen geglättet; Wst. $16.5 \mathrm{~mm}$; Abr: gezackt; Mat.: 3.1; Farbe außen: dunkelbraun (7.5YR4/ 3), Farbe Bruch: dunkelgrau (7.5YR3/1), Farbe innen: dunkelgrau (10YR3/1).

\section{Inv. 2028/97-3.3-4}

(gehört wahrscheinlich zu 228/96-4.1-4)

Schnitt 2/97, US 28

Wandfragmente eines nicht näher bestimmbaren GefäBes

Obfl. außen und innen geglättet; Wst. $16.5 \mathrm{~mm}$; Abr: wenig gezackt; Mat.: 2.2; Farbe außen: hellbraun (5YR6/ 6), Farbe Bruch: braunrot (5YR4/6), Farbe innen: braungelb (7.5YR7/6).

Inv. 2028/97-3.5-73

Schnitt 2/97, US 28

68 Wandfragmente unterschiedlicher, nicht näher bestimmbarer Gefäße.

\section{Inv. 2028/97-6.1}

Schnitt 2/97, US 28

Unretuschierter Abschlag

L 23 mm, B 27 mm, D 14-17 mm; Querschnitt: trapezoid; Farbe: graubraun (7.5YR3/3); veränderte Farbe durch Hitze: dunkelrotbraun (2.5YR4/4).

\section{Inv. 2028/97-6.2}

Schnitt 2/97, US 28

Trümmer

L 26 mm, B $11 \mathrm{~mm}$, D 1-8 mm; Querschnitt: triangulär; veränderte Farbe durch Hitze: rotbraun (2.5YR4/4).

\section{Inv. 2028/97-6.3}

Schnitt 2/97, US 28

Trümmer

L 22 mm, B 17 mm, D 4-6 mm; Querschnitt: sattelförmig; veränderte Farbe durch Hitze: dunkelrotbraun (2.5YR4/4).

\section{Inv. 2028/97-6.4}

Schnitt 2/97, US 28

Abschlagfragment

L $38 \mathrm{~mm}$, B $15 \mathrm{~mm}$, D 5-10 mm; Querschnitt: trapezoid; veränderte Farbe durch Hitze: hellrotbraun (2.5YR6/3).

\section{Inv. 2028/97-6.5}

Schnitt 2/97, US 28

Trümmer

L 29 mm, B 20 mm, D 4-22 mm; Querschnitt: trapezoid; veränderte Farbe durch Hitze: dunkelrotbraun (2.5YR4/ $4)$.

\section{Inv. 2028/97-6.6}

Schnitt 2/97, US 28

Abschlag

L 28 mm, B 15 mm, D 1-9 mm; Querschnitt: trapezoid; Farbe: hellrotbraun (2.5YR6/3).

\section{Inv. 2038/97-10.1}

Schnitt 2/97 US 38

Wandfragment eines nicht näher bestimmbaren Gefäßes Obfl. außen geglättet und innen nicht mehr vorhanden; Wst. $10 \mathrm{~mm}$; Abr.: abgerundet; Mat.: 3.4; Farbe außen: braun (10YR5/4), Farbe Bruch: dunkelbraun (10YR4/3), Farbe innen: braun (10YR5/4).

\section{Inv. 2038/97-10.2}

Schnitt 2/97 US 38

Wandfragment eines nicht näher bestimmbaren Gefäßes Obfl. außen und innen nicht mehr vorhanden; Wst. $8.5 \mathrm{~mm}$; Abr.: abgerundet; Mat.: 2.1; Farbe außen: rot (2.5YR5/8), Farbe Bruch: rotbraun (2.5YR4/4), Farbe innen: $\operatorname{rot}(2.5 \mathrm{YR} 5 / 8)$.

\section{Inv. 2054/97-1.1}

Schnitt 2/97, US 26

Wandfragment eines nicht näher bestimmbaren Gefäßes Obfl. außen und innen nicht mehr vorhanden; Wst. $11.5 \mathrm{~mm}$; Abr.: wenig abgerundet; Mat.: 4.3; Farbe außen: hellrot (2.5YR6/8), Farbe Bruch: dunkelrotbraun (2.5YR3/4), Farbe innen: hellrot (2.5YR6/8).

\section{Inv. 2059/97-3.1}

(gehört wahrscheinlich zu Inv. 228/96-4.7-10)

Schnitt 2/97, US 7

Wandfragment eines nicht näher bestimmbaren Gefäßes Obfl. außen und innen geglättet; Wst. $10 \mathrm{~mm}$; Abr.: gezackt; Mat.: 2.7; Farbe außen: gelbbraun (7.5YR6/6), Farbe Bruch: dunkelbraun (7.5YR4/4), Farbe innen: gelbbraun (7.5YR6/6).

\section{Inv. 2059/97-3.2}

Schnitt 2/97, US 7

Wandfragment eines nicht näher bestimmbaren Gefäßes Obfl. außen und innen nicht mehr vorhanden; Wst. $9 \mathrm{~mm}$; Abr:: abgerundet; Mat.: 2.1; Farbe außen: braungelb (7.5YR6/6), Farbe Bruch: dunkelgelbbraun (7.5YR5/6), Farbe innen: braungelb (7.5YR6/6).

Inv. 2060/97-1.1-3

Schnitt 2/97, US 26

Wandfragmente eines nicht näher bestimmbaren Gefäßes

Obfl. oben geglättet und unten grob geglättet; Wst. $24.5 \mathrm{~mm}$; Abr.: wenig gezackt; Mat.: 4.2; Farbe außen: sehr dunkelgelbbraun (10YR4/6), Farbe Bruch: rotbraun 
(5YR4/4), Farbe innen: sehr dunkelgelbbraun (10YR4/ $6)$.

\section{Inv. 2060/97-1.4-5}

Schnitt 2/97, US 26

Boden eines nicht näher bestimmbaren Gefäßes Obfl. außen und innen geglättet; Wst. 13 mm; Abr.: wenig abgerundet; Mat.: 4.5; Farbe außen: sehr hellorange
(5YR7/8), Farbe Bruch: dunkelorange (5YR5/8), Farbe innen: sehr hellorange (5YR7/8).

\section{Inv. 2060/97-1.6-9}

Schnitt 2/97, US 26

4 Wandfragmente unterschiedlicher, nicht näher bestimmbarer Gefäße.

Dr. Angela Carneiro Institut für Ur- und Frühgeschichte, Universität Wien, Franz Klein-Gasse 1, A-1190 Wien E-Mail: angela.carneiro@univie.ac.at

Abbildungsnachweis: Alle Abb. (C) Velia-Archiv, IKA Wien; Taf. 1-4: Zeichnung Verf., graphische Gestaltung A. Sulzgruber (IKA Wien). 
